\title{
An Energy Stable, Hexagonal Finite Difference Scheme for the 2D Phase Field Crystal Amplitude Equations
}

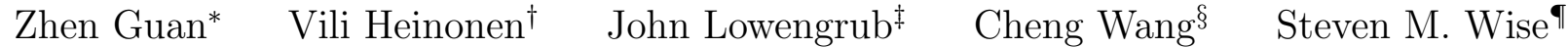

May 19, 2016

\begin{abstract}
In this paper we construct an energy stable finite difference scheme for the amplitude expansion equations for the two-dimensional phase field crystal (PFC) model. The equations are formulated in a periodic hexagonal domain with respect to the reciprocal lattice vectors to achieve a provably unconditionally energy stable and solvable scheme. To our knowledge, this is the first such energy stable scheme for the PFC amplitude equations. The convexity of each part in the amplitude equations is analyzed, in both the semi-discrete and fully-discrete cases. Energy stability is based on a careful convexity analysis for the energy (in both the spatially continuous and discrete cases). As a result, unique solvability and unconditional energy stability are available for the resulting scheme. Moreover, we show that the scheme is point-wise stable for any time and space step sizes. An efficient multigrid solver is devised to solve the scheme, and a few numerical experiments are presented, including grain rotation and shrinkage and grain growth studies, as examples of the strength and robustness of the proposed scheme and solver.
\end{abstract}

Keywords: phase field crystal, amplitude equations, energy stable scheme, hexagonal finite differences, multigrid

\section{Introduction}

One of the major challenges in material science is the complex interplay between macroscopic scale and atomistic scale material properties. For instance, in crystalline materials, liquid-solid and solidsolid phase transitions can be mediated and limited by a larger framework of order and symmetry that includes topological defects, such as dislocations, vacancies, and grain boundaries. But, because of the great number of degrees of freedom involved, simulating crystalline material properties across disparate length and time scales is an enormous obstacle. In this article we concentrate on the popular phase field crystal modeling framework, which can capture phase transition dynamics on mesoscopic time scales, and on length scales that extend from atomic to mesoscopic.

Increases in computational power have given new means to study discrete many-particle systems. One of the most straightforward methods is molecular dynamics (MD) simulation, which is based on explicitly calculating the structure of matter as a function of inter-particle potentials.

\footnotetext{
${ }^{*}$ Department of Mathematics, The University of California, Irvine, CA (zguan2@math.uci.edu)

${ }^{\dagger}$ COMP Centre of Excellence at the Department of Applied Physics, School of Science, Aalto University, Aalto, Finland (vili.heinonen@aalto.fi)

${ }^{\ddagger}$ Department of Mathematics, The University of California, Irvine, CA (lowengrb@math.uci.edu)

${ }^{\S}$ Department of Mathematics, The University of Massachusetts, Dartmouth, MA (cwang1@umassd.edu)

"Department of Mathematics, The University of Tennessee, Knoxville, TN (Corresponding Author: swise@math.utk.edu)
} 
MD simulations can be applied either to solve for the structure evolution in time or to find the minimum energy state of the system using a suitable optimization algorithm. MD methods have been hugely successful in explaining different mesoscopic phenomena, but they come with certain grave limitations. One major disadvantage of the MD simulation is its short time scale, typically the time scale of thermal vibration. Due to this, MD methods are often limited to study relatively fast phenomena. Another problem is simulation data processing: MD methods involve a great deal of information and it turns out to be extremely difficult to separate, analyze, and identify emergent phenomena.

To overcome the aforementioned difficulties we use a coarser statistical field theoretical framework. Instead of studying the positions of single particles, we use the ensemble averaged one-particle probability density field that is calculated by taking the average of the particle positions in a thermal bath. This approach eliminates the time scale of the thermal vibration and allows for extending the analysis to a larger diffusive time scale. In this article we use the PFC framework originally introduced by Elder et al. [11, 12, 13], which is one of the simplest models to tackle a wide range of different problems, including, grain-boundary melting [7, 30] and energy [28]; fractal growth [39]; surface ordering [1, 2, 34, 35]; epitaxial growth [11, 12, 13, 46]; yield stress of polycrystals $[24,37,38]$; and glass transitions $[8,9]$.

PFC models represent crystalline solids by a spatially oscillating, smooth number density field that is very close to the one-mode approximation of the crystal structure. The wavelength is related to the atomic spacing of the crystal, and, therefore, spatial discretization for numerical approximation is constrained to be less than one atomistic distance. However, for the description of a near perfect crystal grain, most of what is required to encode the information about the grain is its orientation, which often varies on a much longer length scale. In a nutshell, this is the idea behind the amplitude expansion framework for the PFC model. The amplitude expansion representation of the PFC model, which we describe in detail below, can be obtained through renormalization of the solid PFC amplitudes [3, 17, 18], or by simpler, heuristic arguments [32]. In the amplitude expansion framework, instead of solving the actual number density field, we solve for the envelope function of the density variation. These complex envelope functions (amplitudes) carry much more information than just grain orientation; they also encode any deformations (not only rotations) and, perhaps more importantly, liquid-solid phase transition, or some other information of local symmetry breaking, e.g., stripe-to-hexagonal transitions. The amplitude expansion framework has been successfully used for studying heteroepitaxial systems [14, 15, 26, 27] and it can be used to make a connection between microscopic and macroscopic descriptions of material allowing for analysis of different time and space scales based on the knowledge at the macroscopic level [23].

The numerical study of the phase field crystal amplitude expansion equations, however, is limited. The are existing numerical implementations are based on finite difference, finite volume, or finite element methods $[4,15,47]$. Adaptive mesh refinement techniques can improve the efficiency of these types of approachs tremendously [4], since the amplitude variables have spatial variation on a wide range of scales. Semi-implicit pseudo-spectral type methods are another popular choice for numerical simulations $[23,36]$, but these cannot be extended to an adaptive framework easily. As we will see the amplitude equations are fourth-order, coupled, highly nonlinear parabolic equations. Explicit methods, therefore, can suffer from severe time step restrictions to avoid numerical instability. Of course, semi-implicit time discretization can help to offset the numerical stiffness present in the amplitude expansion equations, though none of the implementations just mentioned are accompanied by a rigorous stability analysis. There has been, to our knowledge, no numerical analysis of the equations, and we aim to address this deficiency herein.

In this paper we present a semi-implicit, first-order in time, unconditionally energy stable, and unconditionally uniquely solvable scheme for PFC amplitude equations based on the convex 
splitting idea. The framework of convex splitting, popularized by Eyre [16], has been successfully applied to various PFC models [5, 20, 25, 45], but its extension to the present setting is, we will see, rather complicated. Another important feature of our scheme is that we perform the spatial discretization on hexagonal mesh, which has been employed extensively in global climate models $[22,40]$ and elsewhere $[29,50]$. The hexagonal mesh, which brings the added benefit that it is more 'isotropic' than the standard cartesian mesh [31], allows us to create a numerical energy such that our finite difference scheme can be viewed as a discrete variation of this energy, which facilitates our theoretical analysis. Also, as a part of this present we, we devise an efficient nonlinear (geometric) multigrid algorithm to solve the scheme at each time step. For the sake of brevity, we cannot delve too deeply into the complicated convergence analyses of our nonlinear algebraic solver. However, in [50], the author employ a local Fourier analysis to study a geometric multigrid algorithm on a hexagonal, hierarchical mesh, which may be useful for analyzing our own multigrid solver in the future.

The paper is organized as follows. In section 2 we introduce the amplitude equation and the hexagonal discretization. In section 3 the semi- and fully-discrete energy stable schemes are detailed. In section 4 we present numerical experiments verifying properties for both the amplitude equation and the proposed algorithms.

\section{Phase-Field-Crystal (PFC) Amplitude Expansion Model}

In this section we introduce the two-dimensional phase field crystal (PFC) amplitude equations. We start with some basic notations, tools, and terminology from the Fourier analysis on Bravais lattices.

\subsection{Motivation for and Derivation of the Amplitude Equations}

The phase-field-crystal model describes the structure of a system in terms of a coarse-grained atomistic number density field $\rho$. The energy of the system is defined as

$$
E_{\mathrm{pfc}}[\rho]=\int_{\Omega}\left[\frac{B_{0}+B_{4}}{2} \rho^{2}-B_{4}|\nabla \rho|^{2}+\frac{B_{4}}{2}(\Delta \rho)^{2}-\frac{\tau}{3} \rho^{3}+\frac{\nu}{4} \rho^{4}\right] d \boldsymbol{x},
$$

where $B_{0}, B_{4}, \tau$, and $\nu$ are system parameters, and $\Omega$ is a two-dimensional, periodic domain. We will always assume that $B_{4}, \nu>0$. The physical interpretations of the parameters are explained in references $[13,28]$. The dynamics of the PFC model is taken to be a conservative gradient flow, given by

$$
\partial_{t} \rho=\Delta \delta_{\rho} E_{\mathrm{pfc}}=\Delta\left[\left(B_{0}+B_{4}\right) \rho+2 B_{4} \Delta \rho+B_{4} \Delta^{2} \rho-\tau \rho^{2}+\nu \rho^{3}\right],
$$

which in turn leads to a dissipation of the energy $E_{\mathrm{pfc}}$.

For suitable parameter choices, the PFC model predicts the existence of a perfect hexagonal crystal for the equilibrium solid state in two dimensions [32]. The hexagonal/triangular crystal exists as Bravais lattice with lattice vectors

$$
\boldsymbol{a}_{1}=\frac{a_{0}}{2}\left(\sqrt{3} \boldsymbol{e}_{1}+\boldsymbol{e}_{2}\right) \quad \text { and } \quad \boldsymbol{a}_{2}=a_{0} \boldsymbol{e}_{2},
$$

$a_{0}=\frac{4}{\sqrt{3}} \pi$ is the "equilibrium" lattice spacing predicted in the PFC model. Associated to these Bravais lattice vectors are the so-called reciprocal lattice vectors

$$
\boldsymbol{g}_{1}=\frac{4 \pi}{a_{0} \sqrt{3}} \boldsymbol{e}_{1} \quad \text { and } \quad \boldsymbol{g}_{2}=\frac{2 \pi}{a_{0} \sqrt{3}}\left(-\boldsymbol{e}_{1}+\sqrt{3} \boldsymbol{e}_{2}\right),
$$




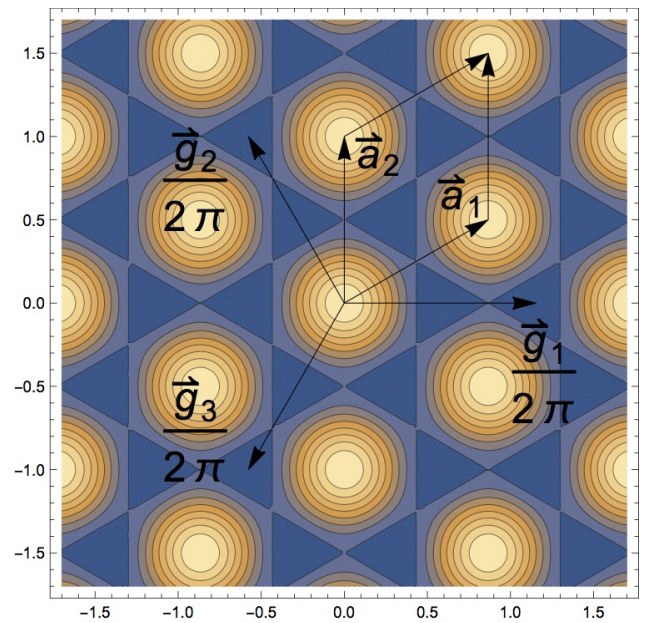

Figure 1: A symmetric one-mode expansion with respect to triangular primitive cell. Here we have chosen the characteristic primitive cell size as $a_{0}=1$ for simplicity. The primitive cell $\mathrm{p}$ is the parallelogram in the upper right of the figure.

which satisfy $\boldsymbol{g}_{1} \cdot \boldsymbol{a}_{j}=2 \pi \delta_{1, j}$ and $\boldsymbol{g}_{2} \cdot \boldsymbol{a}_{j}=2 \pi \delta_{2, j}, j=1,2$. It is often convenient to introduce a third reciprocal lattice vector:

$$
\boldsymbol{g}_{3}=\frac{2 \pi}{a_{0} \sqrt{3}}\left(-\boldsymbol{e}_{1}-\sqrt{3} \boldsymbol{e}_{2}\right)=-\boldsymbol{g}_{1}-\boldsymbol{g}_{2} .
$$

The stress-free equilibrium crystal as represented in the PFC model can be approximated succinctly as the number density

$$
\rho(\boldsymbol{x}, t)=\rho_{0}+\sum_{j=1}^{3} \frac{Q}{4} \exp \left(i \boldsymbol{g}_{j} \cdot \boldsymbol{x}\right)+\sum_{j=1}^{3} \frac{Q}{4} \exp \left(-i \boldsymbol{g}_{j} \cdot \boldsymbol{x}\right),
$$

where $Q \geq 0$ is an amplitude, and $\rho_{0}$ is the average density. This symmetric, "one-mode" Fourier approximation is plotted in Figure 1 together with the lattice and reciprocal lattice vectors. The hexagonal/triangular primitive cell $\mathrm{p}$ is defined as the parallelogram whose bases are $\boldsymbol{a}_{1}$ and $\boldsymbol{a}_{2}$.

Besides this very simple situation (equilibrium solid state), the PFC model can describe several other phase transformation phenomena as well, such as grain boundary formation and motion, solidification, and much more. In all cases, a computational grid is required to capture the atomicscale variation of density $\rho$, which makes the overall computational cost very expensive. To be precise, one must always resolve the lattice spacing $a_{0}$. (The rule-of-thumb is 5-10 grid points per lattice spacing.) On the other hand, we observe that a solid state multi-grain, polycrystalline material, for example, can be roughly described by representing each grain using a one-mode approximation (2.6) with a distinct crystal orientation, i.e., a rotatation angle. The orientation information should only vary with the size of the grain. In other words, to describe many of the features of a PFC solution field $\rho$, one needs information which varies on a larger length scale than the atomic spacing.

To derive the PFC amplitude equations, we consider a more general one-mode approximation using the three complex amplitudes $\eta_{1}, \eta_{2}$ and $\eta_{3}$, which vary in both time and space, and we represent the number density as

$$
\rho(\boldsymbol{x}, t)=\rho_{0}+\sum_{j=1}^{3} \eta_{j}(\boldsymbol{x}, t) \exp \left(i \boldsymbol{g}_{j} \cdot \boldsymbol{x}\right)+\sum_{j=1}^{3} \eta_{j}^{*}(\boldsymbol{x}, t) \exp \left(-i \boldsymbol{g}_{j} \cdot \boldsymbol{x}\right) .
$$


Here $*$ stands for the complex conjugation, and $\rho_{0}$ is a real constant, which again stands for the average of the density field $\rho$. In a more general model, one can allow $\rho_{0}$ to be a function of time and space as well, but we leave this generalization for future work. For the convenience of further analysis, we present the complex amplitudes in the form of separate real and imaginary parts:

$$
\eta_{j}(\boldsymbol{x}, t)=u_{j}(\boldsymbol{x}, t)+i v_{j}(\boldsymbol{x}, t) .
$$

Now, roughly speaking, to track the state of a system described by the PFC model, one could track the time and space variations of the amplitude variables, rather than $\rho$. One would expect that the spatial variations of these amplitudes are on a much larger scale than $a_{0}$, and we will see that this is often the case. We only need now to determine what equations should describe the evolution of the amplitude variables.

To define the amplitude equations, we need a couple of non-standard operators. Subordinate to the reciprocal vectors $(2.4)-(2.5)$, we define the three directional derivatives

$$
\mathcal{D}_{j}:=\boldsymbol{g}_{j} \cdot \nabla
$$

and the complex differential operators

$$
\mathcal{G}_{j}:=\Delta+2 i \mathcal{D}_{j}
$$

Observe that

$$
\Delta \phi=\frac{2}{3} \sum_{j=1}^{3} \mathcal{D}_{j}^{2} \phi .
$$

Then, substituting the one-mode expansion (2.7) into the PFC equation and using a multiple-scale expansion method [32] or renormalization group method [3], one can derive the following PFC amplitude equations:

$$
\begin{aligned}
\dot{u}_{j}= & -\mu_{j} \\
\mu_{j}:= & B_{4}\left(\Delta^{2} u_{j}-2 \Delta\left(\mathcal{D}_{j} v_{j}\right)-2 \mathcal{D}_{j}\left(\Delta v_{j}\right)-4 \mathcal{D}_{j}^{2} u_{j}\right)+\left(B_{0}-2 \tau \rho_{0}+3 \nu \rho_{0}^{2}\right) u_{j} \\
& +3 \nu p_{j} u_{j}+\left(6 \nu \rho_{0}-2 \tau\right)\left(u_{\alpha_{j}} u_{\beta_{j}}-v_{\alpha_{j}} v_{\beta_{j}}\right), \\
\dot{v}_{j}= & -\nu_{j} \\
\nu_{j}:= & B_{4}\left(\Delta^{2} v_{j}+2 \Delta\left(\mathcal{D}_{j} u_{j}\right)+2 \mathcal{D}_{j}\left(\Delta u_{j}\right)-4 \mathcal{D}_{j}^{2} v_{j}\right)+\left(B_{0}-2 \tau \rho_{0}+3 \nu \rho_{0}^{2}\right) v_{j} \\
& +3 \nu p_{j} v_{j}-\left(6 \nu \rho_{0}-2 \tau\right)\left(u_{\alpha_{j}} v_{\beta_{j}}+v_{\alpha_{j}} u_{\beta_{j}}\right),
\end{aligned}
$$

for $j=1,2,3$, where

$$
p_{j}:=2 A^{2}-\left|\eta_{j}\right|^{2}=2 A^{2}-u_{j}^{2}-v_{j}^{2} \geq 0
$$

with

$$
A^{2}:=\sum_{j=1}^{3}\left|\eta_{j}\right|^{2}, \quad \eta_{j}=u_{j}+i v_{j}
$$

and

$$
\alpha_{j}, \beta_{j} \in\{1,2,3\} \backslash\{j\}, \quad \alpha_{j}<\beta_{j} .
$$

Remark 2.1. For this presentation, we have followed the derivation in [32], which produces the dynamical equations first. We still need to supply appropriate boundary conditions for the model, which we do in the next sections. With that task done, we can then show that the system has an energy dissipative structure. Specifically, this system is a non-conserved gradient flow with respect to the energy (2.29). For an alternate development, one can derive the energy (2.29) by performing a multiple-scale expansion analysis directly on the PFC energy (2.1) and, subsequently, derive the dynamical amplitude equations (2.12) - (2.15) as the non-conserved gradient flow of (2.29). 


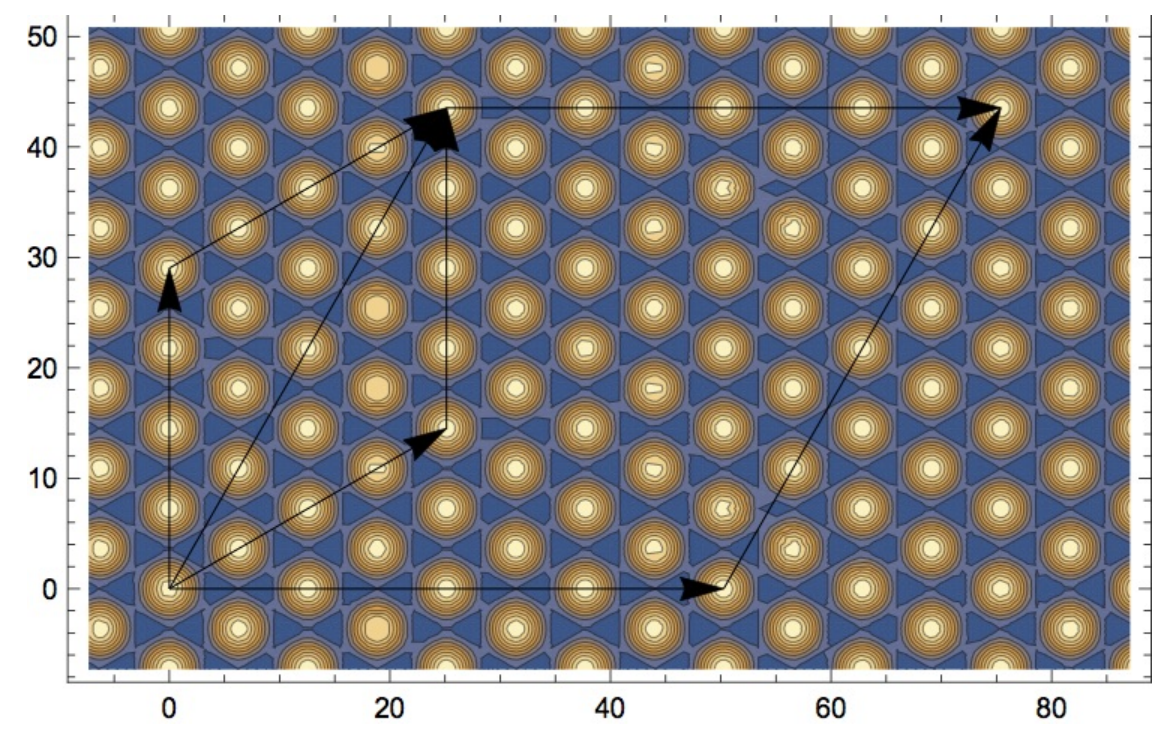

Figure 2: The computational domain D for the PFC amplitude equations. D is the larger parallelogram, defined by setting the domain scale factor $\sigma=4 \sqrt{3} a_{0}$ in Definition 2.3. $\Omega=4 \cdot \mathrm{p}$ is the smaller parallelogram and is exactly as shown in Figure 3. The unrotated crystal (one-mode expansion) is also plotted, as in Figure $3(\theta=0)$. Observe that, with the present choice of $\sigma$, the one-mode approximation of $\rho(2.6)$ is periodic with respect to both $D$ and $\Omega$, which may be desirable and/or useful.

Remark 2.2. There are a couple of important features to observe for this new system of equations. First of all, the amplitude expansion framework seems to hold much promise as a true multi-spatialscale modeling framework, as it can connect PFC (nano meters) to phase field equations. There is a natural coarse-graining mechanism going from the small atomic-scale oscillatory solution to its large-scale amplitude envelope. However, in going to the coarse-grained variables, it appears at first glance to be all for naught. Indeed, the single sixth-order parabolic PFC equation (2.2) has been exchanged for three fourth-order complex parabolic equations for the amplitudes $\eta_{j}$. In practical numerical terms, if the exact same uniform (spatial) mesh resolution is used to solve both problems on the same 'square' $2 D$ domain, this equates to exchanging $3 N^{2}$ (real-valued) degrees of freedom for the PFC problem with $12 N^{2}$ (real-valued) degrees of freedom for the amplitude equations (2.12) - (2.15), where $N$ is the number of grid points along one dimension.

However, in many cases, only half the spatial resolution may be required to resolve the amplitude equations as compared to the $\mathrm{PFC}$ equation. Thus we can adequately resolve the amplitude equations in space using $N / 2$ grid points along one dimension, and the total degrees of freedom work out to be exactly the same in this situation, $3 N^{2}$. But, typically, much less resolution is required for the amplitude equations, perhaps only N/4 or N/8 mesh points along one dimension. More importantly, as with the classical phase field method, the amplitude equations may only need to be resolved in a narrow interfacial band, and thus adaptive mesh techniques are applicable for the complex amplitude equations. On the other hand, adaptive mesh techniques are usually never applicable for the PFC equation, since this has spatially oscillatory solutions.

\subsection{The Amplitude Equations in Hexagonal Domains}

We have some flexibility in what type of periodic domain we choose for our computations involving the amplitude equations. Notice that our formulation of the amplitude model explicitly contains hexagonally anisotropic derivative operators $\mathcal{D}_{j}$. In order to construct a finite difference/finite 
volume method that respects the anisotropies of the problem, we will use a hexagonal/triangular mesh and computational domain, the latter denoted D. Incidentally, it turns out that we can choose D so that it is possible to obtain the stress-free perfect crystal state as a solution to our system of amplitude equations.

Definition 2.3. Let $\boldsymbol{a}_{1}$ and $\boldsymbol{a}_{2}$ be the hexagonal/triangular Bravais vectors. We say that $\mathrm{p} \subset \mathbb{R}^{2}$ is the primitive cell iff it is a parallelogram of the form

$$
\mathrm{p}=\left\{\beta_{1} \boldsymbol{a}_{1}+\beta_{2} \boldsymbol{a}_{2} \in \mathbb{R}^{2} \mid 0<\beta_{i}<1, \quad i=1,2\right\} .
$$

Let $\boldsymbol{g}_{1}$ and $\boldsymbol{g}_{2}$ be reciprocal lattice vectors. We say that $\mathbf{d} \subset \mathbb{R}^{2}$ is the reciprocal cell iff it is a parallelogram of the form

$$
\mathrm{d}=\left\{\beta_{1} \boldsymbol{g}_{1}-\beta_{2} \boldsymbol{g}_{3} \in \mathbb{R}^{2} \mid 0<\beta_{i}<1, \quad i=1,2\right\} .
$$

The computational domain $\mathrm{D} \subset \mathbb{R}^{2}$ is the set $\mathrm{D}=\sigma \cdot \mathrm{d}$, where $\sigma>0$. We say that a function $\phi: \mathbb{R}^{2} \rightarrow \mathbb{C}$ is $\mathrm{D}$-periodic if and only if

$$
\phi\left(\boldsymbol{x}+m_{1} \sigma \boldsymbol{g}_{1}-m_{2} \sigma \boldsymbol{g}_{3}\right)=\phi(\boldsymbol{x}),
$$

for any integer pair $\left(m_{1}, m_{2}\right) \in \mathbb{Z}^{2}$ and all points $\boldsymbol{x} \in \mathbb{R}^{2}$. We say $\phi \in H_{\mathrm{per}}^{m}$ (D) if and only if $\phi \in H_{\mathrm{loc}}^{m}\left(\mathbb{R}^{2}\right)$ and $\phi$ is $\mathrm{D}$-periodic.

We have the following simple result.

Proposition 2.4. Let $\Omega:=M \mathrm{p}$ and $\sigma=M \sqrt{3} a_{0}=4 \pi M$ in Definition 2.3, where $M$ is a positive integer. Suppose that $f: \mathbb{R}^{2} \rightarrow \mathbb{C}$ is $\Omega$-periodic and $g: \mathbb{R}^{2} \rightarrow \mathbb{C}$ is $\mathrm{D}$-periodic. Then $f$ is $\mathrm{D}$-periodic and therefore $f \cdot g$ is $\mathrm{D}$-periodic.

Proof. Suppose that $f: \mathbb{R}^{2} \rightarrow \mathbb{C}$ is $\Omega$-periodic. Then, using $\boldsymbol{g}_{3}=-\boldsymbol{g}_{1}-\boldsymbol{g}_{2}$, and $\boldsymbol{a}_{i} \cdot \boldsymbol{g}_{j}=2 \pi \delta_{i, j}$, $1 \leq i, j \leq 2$, we find, for any integers $m_{1}, m_{2}$,

$$
\begin{aligned}
f(\boldsymbol{x}) & =f\left(\boldsymbol{x}+M m_{1} \boldsymbol{a}_{1}+M m_{2} \boldsymbol{a}_{2}\right) \\
& =f\left(\boldsymbol{x}+4 \pi M\left(\frac{m_{1}-m_{2}}{3}\right) \boldsymbol{g}_{1}-4 \pi M\left(\frac{m_{1}+2 m_{2}}{3}\right) \boldsymbol{g}_{3}\right) .
\end{aligned}
$$

For all integers $m_{1}$ and $m_{2}$ that have the property that $\frac{m_{1}-m_{2}}{3}$ and $\frac{m_{1}+2 m_{2}}{3}$ are also integers, we observe a periodicity with respect to $\mathrm{D}$. For example, for $m_{1}=2, m_{2}=-1$, we have $\frac{m_{1}-m_{2}}{3}=1$ and $\frac{m_{1}+2 m_{2}}{3}=0$. Since the function $\left(\frac{m_{1}-m_{2}}{3}, \frac{m_{1}+2 m_{2}}{3}\right): \mathbb{Z}^{2} \rightarrow \mathbb{R}^{2}$ is onto $\mathbb{Z}^{2}$, the result is proven.

One implication of this last proposition is that, if $\eta_{j}(\boldsymbol{x}, t)$ is $\mathrm{D}$-periodic, with $\mathrm{D}$ chosen as in Proposition 2.4, then $\eta_{j}(\boldsymbol{x}, t) \exp \left(i \boldsymbol{g}_{j} \cdot \boldsymbol{x}\right)$ is D-periodic, since exp $\left(i \boldsymbol{g}_{j} \cdot \boldsymbol{x}\right)$ is $\Omega=M$ p-periodic, for any positive integer $M$. This property may be useful in certain physical scenarios. In particular, the stress-free crystal expressed in (2.6) could be a steady-state solution.

With this type of construction, the computational domain $\mathrm{D}$ is plotted in Figure 2 together with the basic one-mode approximation given in Eq. (2.6). Notice from Figure 2 that D is designed such that its discretization into regular "hexagonal" cells - which will be congruent to both d and D - will be compatible with the reciprocal lattice vectors and the directional derivatives in model. The details for the discretization can be found in section 3 .

We will assume in subsequent sections that the amplitudes $u_{j}, v_{j}, j=1,2,3$, are all D-periodic, without necessarily specifying our choice of $\sigma$. 

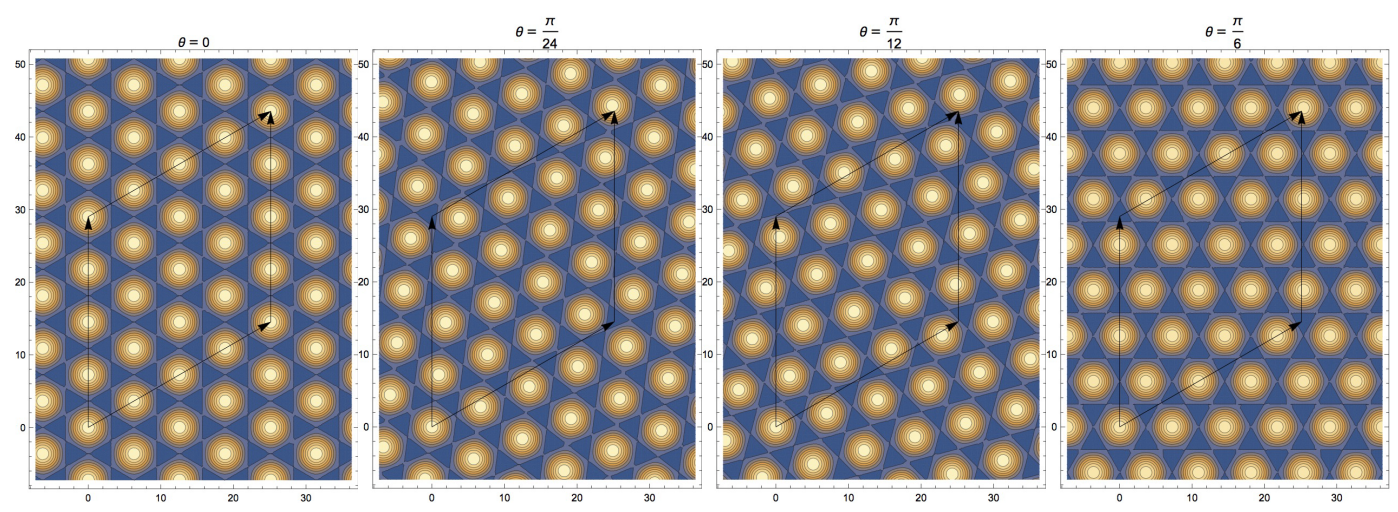

Figure 3: A symmetric triangular one-mode expansion with increasing angles $\theta=0, \frac{\pi}{24}, \frac{\pi}{12}, \frac{\pi}{6}$, rotated clockwise. The parallelogram is the domain $\Omega=4 \mathrm{p}$, which is fixed in space. Clearly, $\theta= \pm \frac{\pi}{6}$ represent the maximum misalignments relative to the unrotated crystal.

\subsection{Crystal Rotations in the Amplitude Expansion Framework}

Complicated arrangements and combinations of the complex amplitudes in (2.7) can be utilized to represent crystals with (nearly) arbitrary orientations, grain boundaries, point defects, et cetera, which capture the real power of the PFC formalism. Here we show how simple crystal rotations are implemented in the amplitude expansion framework. For example, let us introduce a very simple clockwise rotation of a one-mode expansion through an angle $\theta \in[0,2 \pi)$, assuming $\rho_{0}=0$ :

$$
\begin{aligned}
\rho(\boldsymbol{x}, t)= & \sum_{j=1}^{3} \frac{A}{4} \exp \left(i \boldsymbol{g}_{j} \cdot(\mathrm{R}(\theta) \boldsymbol{x}-\boldsymbol{x})\right) \exp \left(i \boldsymbol{g}_{j} \cdot \boldsymbol{x}\right) \\
& +\sum_{j=1}^{3} \frac{A}{4} \exp \left(-i \boldsymbol{g}_{j} \cdot(\mathrm{R}(\theta) \boldsymbol{x}-\boldsymbol{x})\right) \exp \left(-i \boldsymbol{g}_{j} \cdot \boldsymbol{x}\right),
\end{aligned}
$$

where $A>0$ and $\mathrm{R}$ is a rotation matrix

$$
\mathrm{R}(\theta)=\left[\begin{array}{rr}
\cos (\theta) & -\sin (\theta) \\
\sin (\theta) & \cos (\theta)
\end{array}\right]
$$

The effective amplitude functions for the rotated crystal are

$$
\eta_{j}(\boldsymbol{x})=\frac{A}{4} \exp \left(i \boldsymbol{g}_{j} \cdot(\mathrm{R}(\theta) \boldsymbol{x}-\boldsymbol{x})\right), \quad j=1,2,3 .
$$

Note that we are not claiming here that the amplitudes are D-periodic.

See Figures 3 and 4 , where we have assumed $\Omega=4 \mathrm{p}$. The number density $\rho$ is plotted in Figure 3 and the amplitude $u_{1}=\operatorname{Re}\left(\eta_{1}\right)$ is plotted in Figure 4 for increasing rotation angles. One important point is that the amplitudes $\eta_{j}$ and $\rho$ do not share the same periodicities/symmetries, in general. Also observe that the spacial variation of the amplitudes occurs on a larger space scale than that of the number density $\rho$.

\subsection{Energy Dissipation and Convexity Analysis of the Amplitude Equations}

In this section, we show that the amplitude equations dissipate an energy, and we explore some convexity properties of the energy that will help us build an energy stable numerical algorithm for approximating the solutions of the PDE's. First we state an integration-by-parts theorem: 

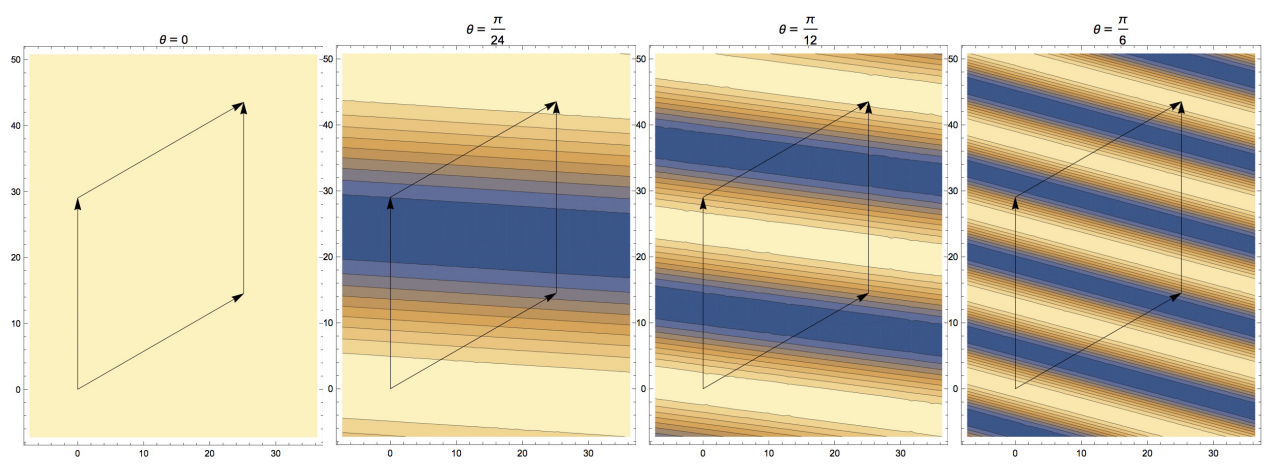

Figure 4: The amplitude $u_{1}$ for a symmetric triangular one-mode expansion rotated clockwise with increasing angles $\theta=0, \frac{\pi}{24}, \frac{\pi}{12}, \frac{\pi}{6}$. The other components of the amplitudes are similar. The parallelogram is the domain $\Omega=4 \mathrm{P}$. The angles $\theta= \pm \frac{\theta}{6}$ represent the maximum misalignments relative to the unrotated crystal. The variations in the amplitudes are expected to occur on a larger spatial scale than those of $\phi$, and the spatial wavelengths of the amplitudes functions $\eta_{j}$ are not expected to be smaller than the case with $\theta=\frac{\pi}{6}$ above.

Proposition 2.5. Suppose that $u, v \in H_{\mathrm{per}}^{1}(\mathrm{D})$. Then

$$
\int_{\mathrm{D}} \mathcal{D}_{j} u(\boldsymbol{x}) v(\boldsymbol{x}) d \boldsymbol{x}=-\int_{\mathrm{D}} u(\boldsymbol{x}) \mathcal{D}_{j} v(\boldsymbol{x}) d \boldsymbol{x} .
$$

More generally, if $u, v \in H_{\mathrm{per}}^{m}(\mathrm{D})$, then

$$
\int_{\mathrm{D}} \mathcal{D}_{j}^{m} u(\boldsymbol{x}) v(\boldsymbol{x}) d \boldsymbol{x}=(-1)^{m} \int_{\mathrm{D}} u(\boldsymbol{x}) \mathcal{D}_{j}^{m} v(\boldsymbol{x}) d \boldsymbol{x} .
$$

Specifically, if $u, v \in H_{\text {per }}^{2}(\mathrm{D})$, we have

$$
\int_{\mathrm{D}} \Delta u(\boldsymbol{x}) v(\boldsymbol{x}) d \boldsymbol{x}=\int_{\mathrm{D}} u(\boldsymbol{x}) \Delta v(\boldsymbol{x}) d \boldsymbol{x}
$$

The PFC amplitude equations may be realized as the gradient flow with respect to an energy, which we now define: for any $\boldsymbol{u}, \boldsymbol{v} \in\left[H_{\text {per }}^{2}(\mathrm{D})\right]^{3}$, set

$$
\begin{aligned}
E(\boldsymbol{u}, \boldsymbol{v}):= & \int_{\mathrm{D}}\left\{\frac{3 \nu}{4}\left(2 A^{4}-\sum_{j=1}^{3}\left|\eta_{j}\right|^{4}\right)+\frac{\nu}{8} \rho_{0}^{4}+6 \nu \rho_{0} \operatorname{Re}\left(\eta_{1} \eta_{2} \eta_{3}\right)\right. \\
& \left.-2 \tau \operatorname{Re}\left(\eta_{1} \eta_{2} \eta_{3}\right)-\frac{\tau}{6} \rho_{0}^{3}+\left(\frac{B_{0}}{2}+\frac{3 \nu \rho_{0}^{2}}{2}-\tau \rho_{0}\right) A^{2}+\frac{B_{4}}{2} \sum_{j=1}^{3}\left|\mathcal{G}_{j} \eta_{j}\right|^{2}\right\} d \boldsymbol{x} .
\end{aligned}
$$

The following dissipation result is valid:

Proposition 2.6. Suppose that $u_{j}, v_{j} \in C^{\infty}\left([0, T] ; C_{\mathrm{per}}^{\infty}(\mathrm{D})\right)$, for $j=1,2,3$, satisfy the PFC amplitude equations $(2.12)-(2.15)$. Then we have the energy dissipation law: for any $t \in[0, T]$,

$$
d_{t} E(\boldsymbol{u}, \boldsymbol{v})=-\sum_{j=1}^{3} \int_{\mathrm{D}}\left\{\mu_{j}^{2}+\nu_{j}^{2}\right\} d \boldsymbol{x} \leq 0,
$$

where $\mu_{j}=\delta_{u_{j}} E(\boldsymbol{u}, \boldsymbol{v})$ and $\nu_{j}=\delta_{v_{j}} E(\boldsymbol{u}, \boldsymbol{v})$ are the variational derivatives of the energy. Equivalently, for any $s \in[0, T]$,

$$
E(\boldsymbol{u}(s), \boldsymbol{v}(s))+\int_{0}^{s}\left\{\sum_{j=1}^{3} \int_{\mathrm{D}}\left\{\mu_{j}^{2}+\nu_{j}^{2}\right\} d \boldsymbol{x}\right\} d t=E(\boldsymbol{u}(0), \boldsymbol{v}(0)) .
$$


Proof. Variational calculations show that $\mu_{j}$ and $\nu_{j}$ are the functional derivatives of $E$ with respect to $u_{j}$ and $v_{j}$, respectively. (We suppress the details for brevity.) The results then follow immediately upon taking the time derivative of the total energy and integrating-by-parts:

$$
\begin{aligned}
d_{t} E(\boldsymbol{u}, \boldsymbol{v})= & \sum_{j=1}^{3} \int_{\mathrm{D}}\left\{B_{4}\left(\Delta^{2} u_{j}-2 \Delta\left(\mathcal{D}_{j} v_{j}\right)-2 \mathcal{D}_{j}\left(\Delta v_{j}\right)-4 \mathcal{D}_{j}^{2} u_{j}\right)+\left(B_{0}-2 \tau \rho_{0}+3 \nu \rho_{0}^{2}\right) u_{j}\right. \\
& \left.+3 \nu p_{j} u_{j}+\left(6 \nu \rho_{0}-2 \tau\right)\left(u_{\alpha_{j}} u_{\beta_{j}}-v_{\alpha_{j}} v_{\beta_{j}}\right)\right\} \dot{u}_{j} d \boldsymbol{x} \\
& +\sum_{j=1}^{3} \int_{\mathrm{D}}\left\{B_{4}\left(\Delta^{2} v_{j}+2 \Delta\left(\mathcal{D}_{j} u_{j}\right)+2 \mathcal{D}_{j}\left(\Delta u_{j}\right)-4 \mathcal{D}_{j}^{2} v_{j}\right)+\left(B_{0}-2 \tau \rho_{0}+3 \nu \rho_{0}^{2}\right) v_{j}\right. \\
& \left.+3 \nu p_{j} v_{j}-\left(6 \nu \rho_{0}-2 \tau\right)\left(u_{\alpha_{j}} v_{\beta_{j}}+u_{\alpha_{j}} v_{\beta_{j}}\right)\right\} \dot{v}_{j} d \boldsymbol{x} .
\end{aligned}
$$

The proof is complete.

For the numerical approximation of dissipative PDE, like the original PFC model and the PFC amplitude equations we examine here, energy stability is an important property, since it often implies norm stabilities that one can use to ultimately bound errors on a theoretical level. The convex splitting paradigm, popularized by Eyre's work [16], is a well-known approach to achieve unconditional numerical energy stability and unconditional unique solvability, at the cost of extra numerical dissipation. The approach has successfully applied to the original PFC equation (2.2) and the modified version; see the related references [5, 6, 10, 19, 21, 25, 42, 45, 48], et cetera. Meanwhile, for the PFC amplitude equations (2.12) - (2.15), there has been no theoretical justification of the unique solvability and energy stability in the existing literature. We focus on this issue now.

The convexity of the energy has to be analyzed for the numerical stability. First we check the convexity of the gradient part.

Proposition 2.7. Suppose that $u_{j}, v_{j} \in H_{\mathrm{per}}^{2}(\mathrm{D})$, for $j=1,2,3$. Then the "gradient" energy, defined as

$$
E_{\mathcal{G}}(\boldsymbol{u}, \boldsymbol{v}):=\frac{1}{2} \sum_{j=1}^{3} \int_{\mathrm{D}}\left|\mathcal{G}_{j} \eta_{j}\right|^{2} d \boldsymbol{x}
$$

is convex.

Proof. We write $E_{\mathcal{G}}(\boldsymbol{u}, \boldsymbol{v})=E_{u}^{\mathcal{G}}(\boldsymbol{u}, \boldsymbol{v})+E_{v}^{\mathcal{G}}(\boldsymbol{u}, \boldsymbol{v})$, where

$$
E_{u}^{\mathcal{G}}(\boldsymbol{u}, \boldsymbol{v}):=\frac{1}{2} \sum_{j=1}^{3} \int_{\mathrm{D}}\left(\Delta u_{j}-2 \mathcal{D}_{j} v_{j}\right)^{2} d \boldsymbol{x}, \quad E_{v}^{\mathcal{G}}(\boldsymbol{u}, \boldsymbol{v}):=\frac{1}{2} \sum_{j=1}^{3} \int_{\mathrm{D}}\left(\Delta v_{j}+2 \mathcal{D}_{j} u_{j}\right)^{2} d \boldsymbol{x} .
$$

Consider the variations $\phi_{j}, \psi_{j} \in H_{\text {per }}^{2}(\mathrm{D})$, for $j=1,2,3$. Then we get

$$
\begin{aligned}
d_{s} E_{u}^{\mathcal{G}}(\boldsymbol{u}+s \boldsymbol{\phi}, \boldsymbol{v}+s \boldsymbol{\psi})= & \sum_{j=1}^{3}\left(\Delta\left(u_{j}+s \phi_{j}\right), \Delta \phi_{j}\right)_{\mathrm{D}}+\sum_{j=1}^{3} 4\left(\mathcal{D}_{j}\left(v_{j}+s \psi_{j}\right), \mathcal{D}_{j} \psi_{j}\right)_{\mathrm{D}} \\
& -\frac{1}{2} \sum_{j=1}^{3}\left\{4\left(\mathcal{D}_{j} \psi_{j}, \Delta\left(u_{j}+s \phi_{j}\right)\right)_{\mathrm{D}}+4\left(\mathcal{D}_{j}\left(v_{j}+s \psi_{j}\right), \Delta \phi_{j}\right)_{\mathrm{D}}\right\}
\end{aligned}
$$


and

$$
\begin{aligned}
d_{s} E_{v}^{\mathcal{G}}(\boldsymbol{u}+s \boldsymbol{\phi}, \boldsymbol{v}+s \boldsymbol{\psi})= & \sum_{j=1}^{3}\left(\Delta\left(v_{j}+s \psi_{j}\right), \Delta \psi_{j}\right)_{\mathrm{D}}+\sum_{j=1}^{3} 4\left(\mathcal{D}_{j}\left(u_{j}+s \phi_{j}\right), \mathcal{D}_{j} \phi_{j}\right)_{\mathrm{D}} \\
& +\frac{1}{2} \sum_{j=1}^{3}\left\{4\left(\mathcal{D}_{j} \phi_{j}, \Delta\left(v_{j}+s \psi_{j}\right)\right)_{\mathrm{D}}+4\left(\mathcal{D}_{j}\left(u_{j}+s \phi_{j}\right), \Delta \psi_{j}\right)_{\mathrm{D}}\right\}
\end{aligned}
$$

As an aside - setting $s=0$, assuming the higher regularities $u_{j}, v_{j} \in H_{\text {per }}^{4}(\mathrm{D})$, and integrating-byparts - we have

$$
\begin{aligned}
\left.d_{s} E_{u}^{\mathcal{G}}(\boldsymbol{u}+s \boldsymbol{\phi}, \boldsymbol{v}+s \boldsymbol{\psi})\right|_{s=0}= & \sum_{j=1}^{3}\left(\Delta^{2} u_{j}, \phi_{j}\right)_{\mathrm{D}}-\sum_{j=1}^{3} 4\left(\mathcal{D}_{j}^{2} v_{j}, \psi_{j}\right)_{\mathrm{D}} \\
& +\sum_{j=1}^{3}\left\{2\left(\mathcal{D}_{j}\left(\Delta u_{j}\right), \psi_{j}\right)_{\mathrm{D}}-2\left(\mathcal{D}_{j}\left(\Delta v_{j}\right), \phi_{j}\right)_{\mathrm{D}}\right\},
\end{aligned}
$$

and

$$
\begin{aligned}
\left.d_{s} E_{v}^{\mathcal{G}}(\boldsymbol{u}+s \boldsymbol{\phi}, \boldsymbol{v}+s \boldsymbol{\psi})\right|_{s=0}= & \sum_{j=1}^{3}\left(\Delta^{2} v_{j}, \psi_{j}\right)_{\mathrm{D}}-\sum_{j=1}^{3} 4\left(\mathcal{D}_{j}^{2} u_{j}, \phi_{j}\right)_{\mathrm{D}} \\
& -\sum_{j=1}^{3}\left\{2\left(\mathcal{D}_{j}\left(\Delta v_{j}\right), \phi_{j}\right)_{\mathrm{D}}-2\left(\mathcal{D}_{j}\left(\Delta u_{j}\right), \psi_{j}\right)_{\mathrm{D}}\right\},
\end{aligned}
$$

which, when taken together, yield the correct contributions to the full variational derivatives of the energy.

The second variations are given by

$$
\begin{aligned}
d_{s}^{2} E_{u}^{\mathcal{G}}(\boldsymbol{u}+s \boldsymbol{\phi}, \boldsymbol{v}+s \boldsymbol{\psi})= & \sum_{j=1}^{3}\left(\Delta \phi_{j}, \Delta \phi_{j}\right)_{\mathrm{D}}+\sum_{j=1}^{3} 4\left(\mathcal{D}_{j} \psi_{j}, \mathcal{D}_{j} \psi_{j}\right)_{\mathrm{D}} \\
& -\frac{1}{2} \sum_{j=1}^{3}\left\{4\left(\mathcal{D}_{j} \psi_{j}, \Delta \phi_{j}\right)_{\mathrm{D}}+4\left(\mathcal{D}_{j} \psi_{j}, \Delta \phi_{j}\right)_{\mathrm{D}}\right\} \\
= & E_{u}^{\mathcal{G}}(\boldsymbol{\phi}, \boldsymbol{\psi}) \geq 0,
\end{aligned}
$$

and

$$
\begin{aligned}
d_{s}^{2} E_{v}^{\mathcal{G}}(\boldsymbol{u}+s \boldsymbol{\phi}, \boldsymbol{v}+s \boldsymbol{\psi})= & \sum_{j=1}^{3}\left(\Delta \psi_{j}, \Delta \psi_{j}\right)_{\mathrm{D}}+\sum_{j=1}^{3} 4\left(\mathcal{D}_{j} \phi_{j}, \mathcal{D}_{j} \phi_{j}\right)_{\mathrm{D}} \\
& +\frac{1}{2} \sum_{j=1}^{3}\left\{4\left(\mathcal{D}_{j} \phi_{j}, \Delta \psi_{j}\right)_{\mathrm{D}}+4\left(\mathcal{D}_{j} \phi_{j}, \Delta \psi_{j}\right)_{\mathrm{D}}\right\} \\
= & E_{v}^{\mathcal{G}}(\boldsymbol{\phi}, \boldsymbol{\psi}) \geq 0 .
\end{aligned}
$$

Therefore, $E_{\mathcal{G}}$ is convex.

Next we discuss the convexity of the non-gradient parts of the energy. 
Lemma 2.8. Let $\beta_{j} \in \mathbb{R}, j=0,1,2$. The fourth-order polynomials $F_{e}, F_{c}: \mathbb{R}^{3} \times \mathbb{R}^{3} \rightarrow \mathbb{R}$, defined via

$$
F_{e}(\boldsymbol{u}, \boldsymbol{v}):=\frac{\beta_{0}}{4}\left(2 A^{4}\right)+\beta_{1} \operatorname{Re}\left(\eta_{1} \eta_{2} \eta_{3}\right)+\frac{\beta_{1}^{2}}{\beta_{0}} A^{2}
$$

and

$$
F_{c}(\boldsymbol{u}, \boldsymbol{v}):=\frac{\beta_{0}}{4}\left(\sum_{j=1}^{3}\left|\eta_{j}\right|^{4}\right)+\beta_{2} A^{2}
$$

are convex, for any $\beta_{1}$, provided that $\beta_{0}>0$ and $\beta_{2} \geq 0$.

Proof. Consider $T_{4}:=\frac{1}{4}\left(2 A^{4}\right)$. The second derivatives of $T_{4}$ become

$$
\frac{\partial^{2} T_{4}}{\partial u_{k} \partial u_{j}}=2 A^{2} \delta_{k, j}+4 u_{k} u_{j}, \quad \frac{\partial^{2} T_{4}}{\partial v_{k} \partial u_{j}}=4 v_{k} u_{j}
$$

and

$$
\frac{\partial^{2} T_{4}}{\partial v_{k} \partial v_{j}}=2 A^{2} \delta_{k, j}+4 v_{k} v_{j}, \quad \frac{\partial^{2} T_{4}}{\partial v_{k} \partial u_{j}}=4 u_{k} v_{j}
$$

The Hessian matrix for this energy density turns out to be

$$
\mathrm{H}_{4}:=2 A^{2} \operatorname{diag}(1,1,1,1,1,1)+4 \mathbf{w} \mathbf{w}^{T},
$$

where $\mathbf{w}:=[\boldsymbol{u}, \boldsymbol{v}]^{T}$. Setting $\mathbf{x}=\left[x_{1}, x_{2}, x_{3}, y_{1}, y_{2}, y_{3}\right]^{T}$ and $z_{j}=x_{j}+i y_{j}$, we have

$$
\mathbf{x}^{T} \mathbf{H}_{4} \mathbf{x}=2 A^{2} \sum_{j=1}^{3}\left(x_{j}^{2}+y_{j}^{2}\right)+4\left(\mathbf{w}^{T} \mathbf{x}\right)^{2}=2 A^{2} \sum_{j=1}^{3}\left|z_{j}\right|^{2}+4\left(\mathbf{w}^{T} \mathbf{x}\right)^{2} \geq 0,
$$

and it follows that $\mathrm{H}_{4}$ is positive semi-definie.

Now, consider $T_{3}:=\operatorname{Re}\left(\eta_{1} \eta_{2} \eta_{3}\right)$. The six partials derivatives are

$$
\frac{\partial T_{3}}{\partial u_{1}}=u_{2} u_{3}-v_{2} v_{3}, \quad \frac{\partial T_{3}}{\partial u_{2}}=u_{1} u_{3}-v_{1} v_{3}, \quad \frac{\partial T_{3}}{\partial u_{3}}=u_{1} u_{2}-v_{1} v_{2},
$$

and

$$
\frac{\partial T_{3}}{\partial v_{1}}=-u_{2} v_{3}-v_{2} u_{3}, \quad \frac{\partial T_{3}}{\partial v_{2}}=-u_{1} v_{3}-v_{1} u_{3}, \quad \frac{\partial T_{3}}{\partial v_{3}}=u_{1} v_{2}-v_{1} u_{2} .
$$

The Hessian of $T_{3}$ is precisely

$$
\mathrm{H}_{3}:=\left[\begin{array}{rrr|rrr}
0 & u_{3} & u_{2} & 0 & -v_{3} & -v_{2} \\
u_{3} & 0 & u_{1} & -v_{3} & 0 & -v_{1} \\
u_{2} & u_{1} & 0 & -v_{2} & -v_{1} & 0 \\
\hline 0 & -v_{3} & -v_{2} & 0 & -u_{3} & -u_{2} \\
-v_{3} & 0 & -v_{1} & -u_{3} & 0 & -u_{1} \\
-v_{2} & -v_{1} & 0 & -u_{2} & -u_{1} & 0
\end{array}\right],
$$

and a short calculation yields

$$
\mathbf{x}^{T} \mathrm{H}_{3} \mathbf{x}=2 \operatorname{Re}\left(z_{1} z_{2} \eta_{3}\right)+2 \operatorname{Re}\left(z_{1} \eta_{2} z_{3}\right)+2 \operatorname{Re}\left(\eta_{1} z_{2} z_{3}\right),
$$

where $z_{j}=x_{j}+i y_{j}, j=1,2,3$. 
The Hessian of $T_{2}:=A^{2}$ is simply

$$
\mathrm{H}_{2}:=2 \operatorname{diag}(1,1,1,1,1,1)=2 \mathrm{I}_{6}
$$

and

$$
\mathbf{x}^{T} \mathrm{H}_{2} \mathbf{x}=2 \sum_{j=1}^{3}\left(x_{j}^{2}+y_{j}^{2}\right)=2 \sum_{j=1}^{3}\left|z_{j}\right|^{2}
$$

The Hessian for $F_{c}$ is

$$
\mathrm{H}_{c}:=\beta_{0} \mathrm{H}_{4}+\beta_{1} \mathrm{H}_{3}+\frac{\beta_{1}^{2}}{\beta_{0}} \mathrm{H}_{2},
$$

and combining the previous calculations together, we have

$$
\begin{aligned}
\mathbf{x}^{T} \mathbf{H}_{c} \mathbf{x}= & \sum_{k=1}^{3} 2\left(\beta_{0} A^{2}+\frac{\beta_{1}^{2}}{\beta_{0}}\right)\left|z_{k}\right|^{2}+2 \beta_{1}\left(\operatorname{Re}\left(z_{1} z_{2} \eta_{3}\right)+\operatorname{Re}\left(z_{1} \eta_{2} z_{3}\right)+\operatorname{Re}\left(\eta_{1} z_{2} z_{3}\right)\right)+4 \beta_{0}\left(\mathbf{w}^{T} \mathbf{x}\right)^{2} \\
= & 2 \beta_{0}\left|z_{1}\right|^{2}\left(\left|\eta_{1}\right|^{2}+\left|\eta_{2}\right|^{2}+\left|\eta_{3}\right|^{2}+\frac{\beta_{1}^{2}}{\beta_{0}^{2}}\right) \\
& +2 \beta_{0}\left|z_{2}\right|^{2}\left(\left|\eta_{1}\right|^{2}+\left|\eta_{2}\right|^{2}+\left|\eta_{3}\right|^{2}+\frac{\beta_{1}^{2}}{\beta_{0}^{2}}\right) \\
& +2 \beta_{0}\left|z_{3}\right|^{2}\left(\left|\eta_{1}\right|^{2}+\left|\eta_{2}\right|^{2}+\left|\eta_{3}\right|^{2}+\frac{\beta_{1}^{2}}{\beta_{0}^{2}}\right) \\
& +2 \beta_{1}\left(\operatorname{Re}\left(z_{1} z_{2} \eta_{3}\right)+\operatorname{Re}\left(z_{1} \eta_{2} z_{3}\right)+\operatorname{Re}\left(\eta_{1} z_{2} z_{3}\right)\right)+4 \beta_{0}\left(\mathbf{w}^{T} \mathbf{x}\right)^{2} \\
= & 2 \beta_{0}\left(\left|z_{1}\right|^{2}+\left|z_{3}\right|^{2}\right)\left|\eta_{2}\right|^{2}+2 \beta_{1} \operatorname{Re}\left(z_{1} \eta_{2} z_{3}\right)+\frac{\beta_{1}^{2}}{\beta_{0}}\left(\left|z_{1}\right|^{2}+\left|z_{3}\right|^{2}\right) \\
& +2 \beta_{0}\left(\left|z_{1}\right|^{2}+\left|z_{2}\right|^{2}\right)\left|\eta_{3}\right|^{2}+2 \beta_{1} \operatorname{Re}\left(z_{1} z_{2} \eta_{3}\right)+\frac{\beta_{1}^{2}}{\beta_{0}}\left(\left|z_{1}\right|^{2}+\left|z_{2}\right|^{2}\right) \\
& +2 \beta_{0}\left(\left|z_{2}\right|^{2}+\left|z_{3}\right|^{2}\right)\left|\eta_{1}\right|^{2}+2 \beta_{1} \operatorname{Re}\left(\eta_{1} z_{2} z_{3}\right)+\frac{\beta_{1}^{2}}{\beta_{0}}\left(\left|z_{2}\right|^{2}+\left|z_{3}\right|^{2}\right) \\
& +2 \beta_{0}\left(\boldsymbol{w}^{T} \boldsymbol{x}\right)^{2}+2 \beta_{0} \sum_{k=1}^{3}\left|\eta_{k}\right|^{2}\left|z_{k}\right|^{2} .
\end{aligned}
$$

Consider

$$
z_{1} z_{2}=\left(x_{1}+i y_{1}\right)\left(x_{2}+i y_{2}\right)=x_{1} x_{2}-y_{1} y_{2}+i\left(y_{1} x_{2}+x_{1} y_{2}\right) .
$$

The following identity is available:

$$
\operatorname{Re}\left(z_{1} z_{2}\right)=x_{1} x_{2}-y_{1} y_{2}, \quad \operatorname{Im}\left(z_{1} z_{2}\right)=y_{1} x_{2}+x_{1} y_{2} .
$$

We now observe the inequality

$$
\left|z_{1}\right|^{2}+\left|z_{2}\right|^{2} \geq 2\left|\operatorname{Re}\left(z_{1} z_{2}\right)\right|
$$

which is equivalent to

$$
x_{1}^{2}+y_{1}^{2}+x_{2}^{2}+y_{2}^{2} \geq \pm 2 x_{1} x_{2} \mp 2 y_{1} y_{2} \text {. }
$$

Similarly, we have

$$
\left|z_{1}\right|^{2}+\left|z_{2}\right|^{2} \geq 2\left|\operatorname{Im}\left(z_{1} z_{2}\right)\right|
$$


which comes from an equivalent inequality

$$
x_{1}^{2}+y_{1}^{2}+x_{2}^{2}+y_{2}^{2} \geq \pm 2 x_{1} y_{2} \pm 2 y_{1} x_{2} .
$$

Now, observe that

$$
\operatorname{Re}\left(z_{1} z_{2} \eta_{3}\right)=\operatorname{Re}\left(z_{1} z_{2}\right) \operatorname{Re}\left(\eta_{3}\right)-\operatorname{Im}\left(z_{1} z_{2}\right) \operatorname{Im}\left(\eta_{3}\right)=\operatorname{Re}\left(z_{1} z_{2}\right) u_{3}-\operatorname{Im}\left(z_{1} z_{2}\right) v_{3}
$$

Putting these facts together, we have

$$
\begin{aligned}
& 2 \beta_{0}\left(\left|z_{1}\right|^{2}+\left|z_{2}\right|^{2}\right)\left|\eta_{3}\right|^{2}+2 \beta_{1} \operatorname{Re}\left(z_{1} z_{2} \eta_{3}\right)+\frac{\beta_{1}^{2}}{\beta_{0}}\left(\left|z_{1}\right|^{2}+\left|z_{2}\right|^{2}\right) \\
& =2 \beta_{0}\left(\left|z_{1}\right|^{2}+\left|z_{2}\right|^{2}\right)\left|\eta_{3}\right|^{2}+2 \beta_{1} \operatorname{Re}\left(z_{1} z_{2}\right) u_{3}-2 \beta_{1} \operatorname{Im}\left(z_{1} z_{2}\right) v_{3}+\frac{\beta_{1}^{2}}{\beta_{0}}\left(\left|z_{1}\right|^{2}+\left|z_{2}\right|^{2}\right) \\
& \geq 2 \beta_{0}\left(\left|z_{1}\right|^{2}+\left|z_{2}\right|^{2}\right)\left|\eta_{3}\right|^{2}-2\left|\beta_{1}\right| \cdot\left|\operatorname{Re}\left(z_{1} z_{2}\right)\right| \cdot\left|u_{3}\right|-2\left|\beta_{1}\right| \cdot\left|\operatorname{Im}\left(z_{1} z_{2}\right)\right| \cdot\left|v_{3}\right|+\frac{\beta_{1}^{2}}{\beta_{0}}\left(\left|z_{1}\right|^{2}+\left|z_{2}\right|^{2}\right) \\
& \geq\left(\left|z_{1}\right|^{2}+\left|z_{2}\right|^{2}\right)\left(2 \beta_{0}\left|\eta_{3}\right|^{2}-2\left|\beta_{1}\right| \cdot\left|u_{3}\right|-2\left|\beta_{1}\right| \cdot\left|v_{3}\right|+\frac{\beta_{1}^{2}}{\beta_{0}}\right) \\
& =\left(\left|z_{1}\right|^{2}+\left|z_{2}\right|^{2}\right)\left(2 \beta_{0} u_{3}^{2}+2 \beta_{0} v_{3}^{2}-2\left|\beta_{1}\right| \cdot\left|u_{3}\right|-2\left|\beta_{1}\right| \cdot\left|v_{3}\right|+\frac{\beta_{1}^{2}}{2 \beta_{0}}+\frac{\beta_{1}^{2}}{2 \beta_{0}}\right) \\
& =\left(\left|z_{1}\right|^{2}+\left|z_{2}\right|^{2}\right)\left(\left(\sqrt{2 \beta_{0}}\left|u_{3}\right|-\sqrt{\frac{\beta_{1}^{2}}{2 \beta_{0}}}\right)^{2}+\left(\sqrt{2 \beta_{0}}\left|v_{3}\right|-\sqrt{\frac{\beta_{1}}{2 \beta_{0}}}\right)^{2}\right) \geq 0 .
\end{aligned}
$$

The other two terms are treated similarly. Therefore, we arrive at

$$
\mathbf{x}^{T} \mathrm{H}_{c} \mathbf{x} \geq 0 .
$$

The proof for $F_{e}(\boldsymbol{u}, \boldsymbol{v})$ similar, in fact, simpler. Observe that

$$
\mathbf{x}^{T} \mathbf{H}_{e} \mathbf{x}=\beta_{0} \sum_{j=1}^{3}\left[\left(u_{j}^{2}+v_{j}^{2}\right)\left(x_{j}^{2}+y_{j}^{2}\right)+2\left(u_{j} x_{j}+v_{j} y_{j}\right)^{2}\right]+2 \beta_{2} \sum_{j=1}^{3}\left|z_{j}\right|^{2} \geq 0,
$$

where $\mathrm{H}_{e}$ is the Hessian of $F_{e}$. The convexity follows immediately.

With the combination of Proposition 2.7 and Lemma 2.8, the following theorem of convexity can be easily established.

Theorem 2.9. Suppose $u_{j}, v_{j} \in H_{\mathrm{per}}^{4}(\mathrm{D}), j=1,2,3$, and define the polynomial energy densities

$$
\begin{aligned}
T_{c}(\boldsymbol{u}, \boldsymbol{v}):= & \frac{3 \nu}{4}\left(2 A^{4}\right)+\left(6 \nu \rho_{0}-2 \tau\right) \operatorname{Re}\left(\eta_{1} \eta_{2} \eta_{3}\right) \\
& +\max \left\{\frac{\left(6 \nu \rho_{0}-2 \tau\right)^{2}}{3 \nu},\left(\frac{B_{0}}{2}+\frac{3 \nu \rho_{0}^{2}}{2}-\tau \rho_{0}\right)\right\} A^{2}
\end{aligned}
$$

and

$$
T_{e}(\boldsymbol{u}, \boldsymbol{v}):=\frac{3 \nu}{4}\left(\sum_{j=1}^{3}\left|\eta_{j}\right|^{4}\right)+\max \left\{0, \frac{\left(6 \nu \rho_{0}-2 \tau\right)^{2}}{3 \nu}-\left(\frac{B_{0}}{2}+\frac{3 \nu \rho_{0}^{2}}{2}-\tau \rho_{0}\right)\right\} A^{2}
$$


Define the energies

$$
E_{c}(\boldsymbol{u}, \boldsymbol{v}):=\int_{\mathrm{D}}\left\{T_{c}(\boldsymbol{u}, \boldsymbol{v})+\frac{B_{4}}{2} \sum_{j=1}^{3}\left|\mathcal{G}_{j} \eta_{j}\right|^{2}\right\} d \boldsymbol{x}, \quad E_{e}(\boldsymbol{u}, \boldsymbol{v}):=\int_{\mathrm{D}} T_{e}(\boldsymbol{u}, \boldsymbol{v}) d \boldsymbol{x} .
$$

Then $E_{c}$ and $E_{e}$ yield a convex splitting of $E$, i.e., $E=E_{c}-E_{e}$, and $E_{c}$ and $E_{e}$ are convex.

We next establish the following coercivity estimate for the energy.

Theorem 2.10. For the energy given by (2.29), the following lower bound is valid:

$$
E(\boldsymbol{u}, \boldsymbol{v}) \geq \gamma_{0} \sum_{j=1}^{3}\left(\left\|u_{j}\right\|_{H^{2}}^{2}+\left\|v_{j}\right\|_{H^{2}}^{2}\right)-\chi_{0},
$$

for any $\boldsymbol{u}, \boldsymbol{v} \in\left[H_{\mathrm{per}}^{2}(\mathrm{D})\right]^{3}$, for some positive constants $\gamma_{0}$ and $\chi_{0}$ that depend on $B, \nu, \tau, B_{4}$ and D.

Proof. For the quadratic diffusion terms, a careful application of the Cauchy inequality shows that

$$
\left(\Delta u_{j}-2 \mathcal{D}_{j} v_{j}\right)^{2} \geq \frac{1}{2}\left(\Delta u_{j}\right)^{2}-4\left|\nabla v_{j}\right|^{2}, \quad 1 \leq j \leq 3
$$

at a point-wise level, using the fact that $\left\|\boldsymbol{g}_{j}\right\|_{2}=1$. This in turn yields

$$
\int_{\mathrm{D}}\left(\Delta u_{j}-2 \mathcal{D}_{j} v_{j}\right)^{2} d \boldsymbol{x} \geq \frac{1}{2}\left\|\Delta u_{j}\right\|_{L^{2}}^{2}-4\left\|\nabla v_{j}\right\|_{L^{2}}^{2}, \quad 1 \leq j \leq 3 .
$$

On the other hand, the term $\left\|\nabla v_{j}\right\|_{L^{2}}^{2}$ can be bounded in the following way:

$$
\left\|\nabla v_{j}\right\|_{L^{2}}^{2}=-\int_{\mathrm{D}} v_{j} \cdot \Delta v_{j} d \boldsymbol{x} \leq\left\|v_{j}\right\|_{L^{2}}\left\|\Delta v_{j}\right\|_{L^{2}} \leq \frac{1}{16}\left\|\Delta v_{j}\right\|_{L^{2}}^{2}+4\left\|v_{j}\right\|_{L^{2}}^{2}, \quad 1 \leq j \leq 3,
$$

with the Cauchy inequality applied at the last step. Consequently, a combination of (2.70) and (2.71) implies that

$$
\int_{\mathrm{D}}\left(\Delta u_{j}-2 \mathcal{D}_{j} v_{j}\right)^{2} d \boldsymbol{x} \geq \frac{1}{2}\left\|\Delta u_{j}\right\|^{2}-\frac{1}{4}\left\|\Delta v_{j}\right\|_{L^{2}}^{2}-16\left\|v_{j}\right\|_{L^{2}}^{2}, \quad 1 \leq j \leq 3 .
$$

A similar estimate may be derived for the other diffusion term:

$$
\int_{\mathrm{D}}\left(\Delta v_{j}+2 \mathcal{D}_{j} u_{j}\right)^{2} d \boldsymbol{x} \geq \frac{1}{2}\left\|\Delta v_{j}\right\|^{2}-\frac{1}{4}\left\|\Delta u_{j}\right\|_{L^{2}}^{2}-16\left\|u_{j}\right\|_{L^{2}}^{2}, \quad 1 \leq j \leq 3 .
$$

Therefore, we arrive at

$$
\begin{aligned}
& \frac{B_{4}}{2} \sum_{j=1}^{3} \int_{\mathrm{D}}\left(\left(\Delta u_{j}-2 \mathcal{D}_{j} v_{j}\right)^{2}+\left(\Delta v_{j}+2 \mathcal{D}_{j} u_{j}\right)^{2}\right) d \boldsymbol{x} \geq \frac{B_{4}}{8} \sum_{j=1}^{3}\left(\left\|\Delta u_{j}\right\|_{L^{2}}^{2}+\left\|\Delta v_{j}\right\|_{L^{2}}^{2}\right) \\
& -8 B_{4} \sum_{j=1}^{3}\left(\left\|u_{j}\right\|_{L^{2}}^{2}+\left\|v_{j}\right\|_{L^{2}}^{2}\right)
\end{aligned}
$$


An application of a simple quadratic inequality gives

$$
8 B_{4} u_{j}^{2} \leq \frac{\nu}{8} u_{j}^{4}+C_{1}, \quad 8 B_{4} v_{j}^{2} \leq \frac{\nu}{8} v_{j}^{4}+C_{1}, \quad C_{1}:=\frac{128 B_{4}^{2}}{\nu} .
$$

Integrating the above inequalities, and substituting into (2.74), we get

$$
\begin{aligned}
\frac{B_{4}}{2} \sum_{j=1}^{3} \int_{\mathrm{D}}\left(\left(\Delta u_{j}-2 \mathcal{D}_{j} v_{j}\right)^{2}+\left(\Delta v_{j}+2 \mathcal{D}_{j} u_{j}\right)^{2}\right) d \boldsymbol{x} \geq & \frac{B_{4}}{8} \sum_{j=1}^{3}\left(\left\|\Delta u_{j}\right\|_{L^{2}}^{2}+\left\|\Delta v_{j}\right\|_{L^{2}}^{2}\right) \\
& -\frac{\nu}{8} \sum_{j=1}^{3}\left(\left\|u_{j}\right\|_{L^{4}}^{4}+\left\|v_{j}\right\|_{L^{4}}^{4}\right) \\
& -6 C_{1}|\mathrm{D}| .
\end{aligned}
$$

For the third-order polynomial terms in (2.29), we apply Young's inequality and observe that

$$
\pm|a b c| \leq \frac{1}{3}\left(|a|^{3}+|b|^{3}+|c|^{3}\right) \leq \frac{\nu}{32\left|6 \nu \rho_{0}-2 \tau\right|}\left(a^{4}+b^{4}+c^{4}\right)+C_{2},
$$

where $C_{2}>0$ depends upon $\nu>0, \rho_{0}$ and $\tau$. Applying this last inequality, we find

$$
\begin{aligned}
& \left(6 \nu \rho_{0}-2 \tau\right) \int_{\mathrm{D}}\left(u_{1} u_{2} u_{3}-u_{1} v_{2} v_{3}-v_{1} u_{2} v_{3}-v_{1} v_{2} u_{3}\right) d \boldsymbol{x} \\
& \geq-\frac{\nu}{8} \sum_{j=1}^{3}\left(\left\|u_{j}\right\|_{L^{4}}^{4}+\left\|v_{j}\right\|_{L^{4}}^{4}\right)-4\left|6 \nu \rho_{0}-2 \tau\right| C_{2}|\mathrm{D}| .
\end{aligned}
$$

Meanwhile, for the fourth order polynomial terms appearing in (2.29), the following estimate is straightforward:

$$
\frac{3 \nu}{2}\left(\sum_{j=1}^{3}\left(u_{j}^{2}+v_{j}^{2}\right)\right)^{2}-\frac{3 \nu}{4} \sum_{j=1}^{3}\left(u_{j}^{2}+v_{j}^{2}\right)^{2} \geq \frac{3 \nu}{4} \sum_{j=1}^{3}\left(u_{j}^{4}+v_{j}^{4}\right),
$$

which in turn leads to

$$
\int_{\mathrm{D}}\left\{\frac{3 \nu}{2}\left(\sum_{j=1}^{3}\left(u_{j}^{2}+v_{j}^{2}\right)\right)^{2}-\frac{3 \nu}{4} \sum_{j=1}^{3}\left(u_{j}^{2}+v_{j}^{2}\right)^{2}\right\} d \boldsymbol{x} \geq \frac{3 \nu}{4} \sum_{j=1}^{3}\left(\left\|u_{j}\right\|_{L^{4}}^{4}+\left\|v_{j}\right\|_{L^{4}}^{4}\right) .
$$

The combination of $(2.76),(2.78)$ and $(2.80)$, as well as the fact that one or both of $B_{0}$ and $\tau$ may be negative, gives the following lower bound for the energy:

$$
\begin{aligned}
E(\boldsymbol{u}, \boldsymbol{v}) \geq & \left(\frac{B_{0}}{2}+\frac{3 \nu \rho_{0}^{2}}{2}-\tau \rho_{0}\right) \sum_{j=1}^{3}\left(\left\|u_{j}\right\|_{L^{2}}^{2}+\left\|v_{j}\right\|_{L^{2}}^{2}\right)+\frac{B_{4}}{8} \sum_{j=1}^{3}\left(\left\|\Delta u_{j}\right\|_{L^{2}}^{2}+\left\|\Delta v_{j}\right\|_{L^{2}}^{2}\right) \\
& +\frac{\nu}{2} \sum_{j=1}^{3}\left(\left\|u_{j}\right\|_{L^{4}}^{4}+\left\|v_{j}\right\|_{L^{4}}^{4}\right)-\left(6 C_{1}+8 \tau C_{2}\right)|\mathrm{D}| \\
\geq & -\left|\frac{B_{0}}{2}+\frac{3 \nu \rho_{0}^{2}}{2}-\tau \rho_{0}\right| \sum_{j=1}^{3}\left(\left\|u_{j}\right\|_{L^{2}}^{2}+\left\|v_{j}\right\|_{L^{2}}^{2}\right)+\frac{B_{4}}{8} \sum_{j=1}^{3}\left(\left\|\Delta u_{j}\right\|_{L^{2}}^{2}+\left\|\Delta v_{j}\right\|_{L^{2}}^{2}\right) \\
& +\frac{\nu}{2} \sum_{j=1}^{3}\left(\left\|u_{j}\right\|_{L^{4}}^{4}+\left\|v_{j}\right\|_{L^{4}}^{4}\right)-\left(6 C_{1}+4\left|6 \nu \rho_{0}-2 \tau\right| C_{2}\right)|\mathrm{D}| .
\end{aligned}
$$


Now, there is a constant $C_{3} \geq 0$ that depends upon the parameters $B_{0}, \tau, \rho_{0} \in \mathbb{R}$ and $B_{4}, \nu>0$ such that

$$
\frac{\nu}{2} u_{j}^{4}-\left|\frac{B_{0}}{2}+\frac{3 \nu \rho_{0}^{2}}{2}-\tau \rho_{0}\right| u_{j}^{2} \geq \frac{B_{4}}{8} u_{j}^{2}-C_{3}, \quad j=1,2,3
$$

and likewise for $v_{j}$. Therefore,

$$
\begin{aligned}
\frac{\nu}{2} \sum_{j=1}^{3}\left(\left\|u_{j}\right\|_{L^{4}}^{4}+\left\|v_{j}\right\|_{L^{4}}^{4}\right) & -\left|\frac{B_{0}}{2}+\frac{3 \nu \rho_{0}^{2}}{2}-\tau \rho_{0}\right| \sum_{j=1}^{3}\left(\left\|u_{j}\right\|_{L^{2}}^{2}+\left\|v_{j}\right\|_{L^{2}}^{2}\right) \\
& \geq \frac{B_{4}}{8} \sum_{j=1}^{3}\left(\left\|u_{j}\right\|_{L^{2}}^{2}+\left\|v_{j}\right\|_{L^{2}}^{2}\right)-6 C_{3}|\mathrm{D}| .
\end{aligned}
$$

Now we need an elliptic regularity result:

$$
\left\|u_{j}\right\|_{H^{2}}^{2} \leq C_{4}\left(\left\|u_{j}\right\|^{2}+\left\|\Delta u_{j}\right\|^{2}\right), \quad\left\|v_{j}\right\|_{H^{2}}^{2} \leq C_{4}\left(\left\|v_{j}\right\|^{2}+\left\|\Delta v_{j}\right\|^{2}\right), \quad 1 \leq j \leq 3
$$

where $C_{4}>0$ only depends on D. This can be proven using Fourier analysis on the parallelogram D. Therefore,

$$
\begin{aligned}
E(\boldsymbol{u}, \boldsymbol{v}) & \geq \frac{B_{4}}{8} \sum_{j=1}^{3}\left(\left\|u_{j}\right\|_{L^{2}}^{2}+\left\|\Delta u_{j}\right\|_{L^{2}}^{2}+\left\|v_{j}\right\|_{L^{2}}^{2}+\left\|\Delta v_{j}\right\|_{L^{2}}^{2}\right)-\left(6 C_{1}+6 C_{3}+4\left|6 \nu \rho_{0}-2 \tau\right| C_{2}\right)|\mathrm{D}| \\
& \geq \frac{B_{4}}{8 C_{4}} \sum_{j=1}^{3}\left(\left\|u_{j}\right\|_{H^{2}}^{2}+\left\|v_{j}\right\|_{H^{2}}^{2}\right)-\left(6 C_{1}+6 C_{3}+4\left|6 \nu \rho_{0}-2 \tau\right| C_{2}\right)|\mathrm{D}| .
\end{aligned}
$$

The proof for Theorem 2.10 is complete.

\section{Energy Stable Algorithms for the Amplitude Equation}

In this section we present a first-order accurate (in time) energy stable scheme, in both the spacecontinuous and fully discrete cases.

\subsection{A Space-Continuous Energy Stable Scheme}

We begin with a space-continuous (semi-discrete) energy stable scheme for the amplitude equations that takes advantage of the convex splitting of the energy that we have introduced. For this purpose, we need an extension of a theorem given in [45].

Theorem 3.1. Suppose that $\phi, \psi \in\left[H_{\mathrm{per}}^{4}(\mathrm{D})\right]^{6}$. Consider a convex splitting of the energy $E$ of the type given in the last result. Then

$$
E(\boldsymbol{\phi})-E(\boldsymbol{\psi}) \leq\left(\boldsymbol{\delta} E_{c}(\boldsymbol{\phi})-\boldsymbol{\delta} E_{e}(\boldsymbol{\psi}), \boldsymbol{\phi}-\boldsymbol{\psi}\right)_{L^{2}(\mathrm{D})},
$$

where $\boldsymbol{\delta}$ denotes the variational derivative.

Proof. Let $E_{c}(\boldsymbol{\phi})=\int_{\mathrm{D}} e_{c}\left(\boldsymbol{\phi}, \boldsymbol{\phi}_{1}, \boldsymbol{\phi}_{2}, \boldsymbol{\phi}_{3}, \Delta \boldsymbol{\phi}\right) d \boldsymbol{x}$, where $\boldsymbol{\phi}_{j}:=\mathcal{D}_{j} \boldsymbol{\phi}, j=1,2,3$. Since $e_{c}: \mathbb{R}^{30} \rightarrow \mathbb{R}$ is smooth and convex in its arguments, we have

$$
e_{c}(\boldsymbol{q})-e_{c}(\boldsymbol{p}) \geq \nabla_{\boldsymbol{p}} e_{c}(\boldsymbol{p}) \cdot(\boldsymbol{q}-\boldsymbol{p})
$$


for any $\boldsymbol{p}, \boldsymbol{q} \in \mathbb{R}^{30}$. Setting $\boldsymbol{p}=\left(\boldsymbol{\phi}, \boldsymbol{\phi}_{1}, \boldsymbol{\phi}_{2}, \boldsymbol{\phi}_{3}, \Delta \boldsymbol{\phi}\right)$ and $\boldsymbol{q}=\left(\boldsymbol{\psi}, \boldsymbol{\psi}_{1}, \boldsymbol{\psi}_{2}, \boldsymbol{\psi}_{3}, \Delta \boldsymbol{\psi}\right)$, and integrating (3.2), we get

$$
\begin{aligned}
E_{c}(\boldsymbol{\psi})-E_{c}(\boldsymbol{\phi}) \geq & \int_{\mathrm{D}}\left\{\frac{\partial}{\partial \phi} e_{c}\left(\boldsymbol{\phi}, \boldsymbol{\phi}_{1}, \boldsymbol{\phi}_{2}, \boldsymbol{\phi}_{3}, \Delta \boldsymbol{\phi}\right) \cdot(\boldsymbol{\psi}-\boldsymbol{\phi})\right. \\
& +\frac{\partial}{\partial \phi_{1}} e_{c}\left(\boldsymbol{\phi}, \boldsymbol{\phi}_{1}, \boldsymbol{\phi}_{2}, \boldsymbol{\phi}_{3}, \Delta \boldsymbol{\phi}\right) \cdot\left(\boldsymbol{\psi}_{1}-\boldsymbol{\phi}_{1}\right) \\
& +\frac{\partial}{\partial \phi_{2}} e_{c}\left(\boldsymbol{\phi}, \boldsymbol{\phi}_{1}, \boldsymbol{\phi}_{2}, \boldsymbol{\phi}_{3}, \Delta \boldsymbol{\phi}\right) \cdot\left(\boldsymbol{\psi}_{2}-\boldsymbol{\phi}_{2}\right) \\
& +\frac{\partial}{\partial \phi_{3}} e_{c}\left(\boldsymbol{\phi}, \boldsymbol{\phi}_{1}, \boldsymbol{\phi}_{2}, \boldsymbol{\phi}_{3}, \Delta \boldsymbol{\phi}\right) \cdot\left(\boldsymbol{\psi}_{3}-\boldsymbol{\phi}_{3}\right) \\
& \left.+\frac{\partial}{\partial \Delta \phi} e_{c}\left(\boldsymbol{\phi}, \boldsymbol{\phi}_{1}, \boldsymbol{\phi}_{2}, \boldsymbol{\phi}_{3}, \Delta \boldsymbol{\phi}\right) \cdot(\Delta \boldsymbol{\psi}-\Delta \boldsymbol{\phi})\right\} d \boldsymbol{x}
\end{aligned}
$$

Integration-by-parts leads to the following inequality

$$
E_{c}(\boldsymbol{\psi})-E_{c}(\boldsymbol{\phi}) \geq\left(\boldsymbol{\delta} E_{c}(\phi), \boldsymbol{\psi}-\boldsymbol{\phi}\right)_{L^{2}(\mathrm{D})}
$$

By a similar analysis on $E_{e}$, but reversing the roles of $\phi$ and $\boldsymbol{\psi}$, we have

$$
E_{e}(\boldsymbol{\phi})-E_{e}(\boldsymbol{\psi}) \geq\left(\boldsymbol{\delta} E_{e}(\boldsymbol{\psi}), \boldsymbol{\phi}-\boldsymbol{\psi}\right)_{L^{2}(\mathrm{D})}
$$

Adding (3.4) and (3.5) yields

$$
\begin{aligned}
E(\boldsymbol{\psi})-E(\boldsymbol{\phi}) & =E_{c}(\boldsymbol{\psi})-E_{e}(\boldsymbol{\psi})-\left(E_{c}(\boldsymbol{\phi})-E_{e}(\boldsymbol{\phi})\right) \\
& \geq\left(\boldsymbol{\delta} E_{c}(\boldsymbol{\phi}), \boldsymbol{\psi}-\boldsymbol{\phi}\right)_{L^{2}(\mathrm{D})}+\left(\boldsymbol{\delta} E_{e}(\boldsymbol{\psi}), \boldsymbol{\phi}-\boldsymbol{\psi}\right)_{L^{2}(\mathrm{D})} \\
& =\left(\boldsymbol{\delta} E_{c}(\boldsymbol{\phi})-\boldsymbol{\delta} E_{e}(\boldsymbol{\psi}), \boldsymbol{\psi}-\boldsymbol{\phi}\right)_{L^{2}(\mathrm{D})} .
\end{aligned}
$$

Based on our preliminary calculations we can formulate the following (first-order in time) energy 
stable convex splitting scheme: for $j=1,2,3$,

$$
\begin{aligned}
u_{j}^{m+1}-u_{j}^{m}= & -s \mu_{j}^{m+1}, \\
\mu_{j}^{m+1}= & B_{4}\left(\Delta^{2} u_{j}^{m+1}-4 \mathcal{D}_{j}\left(\Delta v_{j}^{m+1}\right)-4 \mathcal{D}_{j}^{2} u_{j}^{m+1}\right) \\
& +2 \max \left\{\frac{\left(6 \nu \rho_{0}-2 \tau\right)^{2}}{3 \nu},\left(\frac{B_{0}}{2}+\frac{3 \nu \rho_{0}^{2}}{2}-\tau \rho_{0}\right)\right\} u_{j}^{m+1} \\
& +6 \nu\left(A^{m+1}\right)^{2} u_{j}^{m+1}-3 \nu\left(\left(u_{j}^{m}\right)^{2}+\left(v_{j}^{m}\right)^{2}\right) u_{j}^{m} \\
& +\left(6 \nu \rho_{0}-2 \tau\right)\left(u_{\alpha_{j}}^{m+1} u_{\beta_{j}}^{m+1}-v_{\alpha_{j}}^{m+1} v_{\beta_{j}}^{m+1}\right) \\
& -2 \max \left\{0, \frac{\left(6 \nu \rho_{0}-2 \tau\right)^{2}}{3 \nu}-\left(\frac{B_{0}}{2}+\frac{3 \nu \rho_{0}^{2}}{2}-\tau \rho_{0}\right)\right\} u_{j}^{m}, \\
v_{j}^{m+1}-v_{j}^{m}= & -s \nu_{j}^{m+1}, \\
\nu_{j}^{m+1}= & B_{4}\left(\Delta^{2} v_{j}^{m+1}+4 \mathcal{D}_{j}\left(\Delta u_{j}^{m+1}\right)-4 \mathcal{D}_{j}^{2} v_{j}^{m+1}\right) \\
& +2 \max \left\{\frac{\left(6 \nu \rho_{0}-2 \tau\right)^{2}}{3 \nu},\left(\frac{B_{0}}{2}+\frac{3 \nu \rho_{0}^{2}}{2}-\tau \rho_{0}\right)\right\} v_{j}^{m+1} \\
& +6 \nu\left(A^{m+1}\right)^{2} v_{j}^{m+1}-3 \nu\left(\left(u_{j}^{m}\right)^{2}+\left(v_{j}^{m}\right)^{2}\right) \boldsymbol{v}_{j}^{m} \\
& -\left(6 \nu \rho_{0}-2 \tau\right)\left(u_{\alpha_{j}}^{m+1} v_{\beta_{j}}^{m+1}+v_{\alpha_{j}}^{m+1} u_{\beta_{j}}^{m+1}\right) \\
& -2 \max \left\{0, \frac{\left(6 \nu \rho_{0}-2 \tau\right)^{2}}{3 \nu}-\left(\frac{B_{0}}{2}+\frac{3 \nu \rho_{0}^{2}}{2}-\tau \rho_{0}\right)\right\} v_{j}^{m} .
\end{aligned}
$$

Observe that, precisely,

$$
\begin{aligned}
\mu_{j} & =\delta_{u_{j}} E_{c}\left(\boldsymbol{u}^{m+1}, \boldsymbol{v}^{m+1}\right)-\delta_{u_{j}} E_{e}\left(\boldsymbol{u}^{m}, \boldsymbol{v}^{m}\right), \\
\nu_{j} & =\delta_{v_{j}} E_{c}\left(\boldsymbol{u}^{m+1}, \boldsymbol{v}^{m+1}\right)-\delta_{v_{j}} E_{e}\left(\boldsymbol{u}^{m}, \boldsymbol{v}^{m}\right) .
\end{aligned}
$$

Thus, invoking Theorems 2.9 and 3.1, we have

Theorem 3.2. Let $\boldsymbol{u}^{m+1}, \boldsymbol{u}^{m}, \boldsymbol{v}^{m+1}, \boldsymbol{v}^{m} \in\left[H_{\mathrm{per}}^{4}(\mathrm{D})\right]^{3}$ be solutions of the scheme (3.7) - (3.10). Then

$$
E\left(\boldsymbol{u}^{m+1}, \boldsymbol{v}^{m+1}\right)+s\left\|\boldsymbol{\mu}^{m+1}\right\|_{L^{2}}^{2}+s\left\|\boldsymbol{\nu}^{m+1}\right\|_{L^{2}}^{2} \leq E\left(\boldsymbol{u}^{m}, \boldsymbol{v}^{m}\right) .
$$

Using the lower bound for the energy given in Theorem 2.10 and the last result, we conclude that

Corollary 3.3. Let $\boldsymbol{u}^{m}, \boldsymbol{v}^{m} \in\left[H_{\mathrm{per}}^{4}(\mathrm{D})\right]^{3}, m=1, \ldots, M$, be the solutions of the scheme (3.7) (3.10) with initial data $\boldsymbol{u}^{0}, \boldsymbol{v}^{0} \in\left[H_{\mathrm{per}}^{2}(\mathrm{D})\right]^{3}$. Then we have

$$
\max _{1 \leq m \leq M}\left(\gamma_{0} \sum_{j=1}^{3}\left(\left\|u_{j}^{m}\right\|_{H^{2}}^{2}+\left\|v_{j}^{m}\right\|_{H^{2}}^{2}\right)\right)+s \sum_{m=1}^{M}\left(\left\|\boldsymbol{\mu}^{m}\right\|_{L^{2}}^{2}+\left\|\boldsymbol{\nu}^{m}\right\|_{L^{2}}^{2}\right) \leq E\left(\boldsymbol{u}^{0}, \boldsymbol{v}^{0}\right)+\chi_{0} .
$$

\subsection{A Fully-Discrete Convex-Splitting Scheme}

In this section we introduce a finite difference discretization of the spatial derivative operators $\mathcal{D}_{j}$ and $\mathcal{G}_{j}$ on the parallelogram D. The mesh size $h$ is given by $h=\frac{\sigma}{n}$, for some positive integer $n$. We define the set of cell center points as

$$
\mathrm{C}:=\left\{\boldsymbol{x}_{k, \ell}=\left(x_{k}, y_{\ell}\right)=h \cdot\left(k-\frac{1}{2}\right) \cdot \boldsymbol{g}_{1}-h \cdot\left(\ell-\frac{1}{2}\right) \cdot \boldsymbol{g}_{3} \mid k, \ell \in \mathbb{Z}\right\} .
$$


The points of $E^{1}, E^{2}$ and $E^{3}$ are defined, respectively, via

$$
\begin{aligned}
& \mathrm{E}^{1}:=\left\{\boldsymbol{x}_{k+\frac{1}{2}, \ell}=\left(x_{k+\frac{1}{2}}, y_{\ell}\right)=h \cdot k \cdot \boldsymbol{g}_{1}-h \cdot\left(\ell-\frac{1}{2}\right) \cdot \boldsymbol{g}_{3} \mid k, \ell \in \mathbb{Z}\right\}, \\
& \mathrm{E}^{2}:=\left\{\boldsymbol{x}_{k+\frac{1}{2}, \ell+\frac{1}{2}}=\left(x_{k+\frac{1}{2}}, y_{\ell+\frac{1}{2}}\right)=h \cdot k \cdot \boldsymbol{g}_{1}-h \cdot \ell \cdot \boldsymbol{g}_{3} \mid k, \ell \in \mathbb{Z}\right\}, \\
& \mathrm{E}^{3}:=\left\{\boldsymbol{x}_{k, \ell+\frac{1}{2}}=\left(x_{k}, y_{\ell+\frac{1}{2}}\right)=h \cdot\left(k-\frac{1}{2}\right) \cdot \boldsymbol{g}_{1}-h \cdot \ell \cdot \boldsymbol{g}_{3} \mid k, \ell \in \mathbb{Z}\right\} .
\end{aligned}
$$

Define the space of cell centered grid functions as $\mathcal{C}:=\{\phi: \mathcal{C} \rightarrow \mathbb{R}\}$. By $\mathcal{C}_{\text {per }} \subset \mathcal{C}$ we denote the linear subspace of $\mathrm{D}$-periodic cell centered functions. Similarly, define the edge centered grid function space $\mathcal{E}^{j}:=\left\{f: \mathrm{E}^{j} \rightarrow \mathbb{R}\right\}$, for $j=1,2,3$. By $\mathcal{E}_{\text {per }}^{j} \subset \mathcal{E}^{j}$ we denote the appropriate linear subspace of D-periodic edge centered functions. We use the notation, $f_{k+\frac{1}{2}, \ell}:=f\left(\boldsymbol{x}_{k+\frac{1}{2}, \ell}\right)$, et cetera. Figure 5 demonstrates such a discretization.

We define the cell-centered inner product as follows: for $\phi, \psi \in \mathcal{C}_{\text {per }}$,

$$
(\phi \| \psi):=\frac{\sqrt{3} h^{2}}{2} \sum_{k=1}^{n} \sum_{\ell=1}^{n} \phi_{k, \ell} \psi_{k, \ell} .
$$

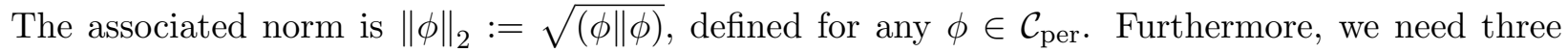
edge-centered inner products:

$$
\begin{aligned}
& {[u \| v]_{1}=\frac{\sqrt{3} h^{2}}{4} \sum_{k=1}^{n} \sum_{\ell=1}^{n}\left\{u_{k+\frac{1}{2}, \ell} v_{k+\frac{1}{2}, \ell}+u_{k-\frac{1}{2}, \ell} v_{k-\frac{1}{2}, \ell}\right\}, \quad \text { for } u, v \in \mathcal{E}_{\mathrm{per}}^{1},} \\
& {[u \| v]_{2}=\frac{\sqrt{3} h^{2}}{4} \sum_{k=1}^{n} \sum_{\ell=1}^{n}\left\{u_{k-\frac{1}{2}, \ell+\frac{1}{2}} v_{k-\frac{1}{2}, \ell+\frac{1}{2}}+u_{k+\frac{1}{2}, \ell-\frac{1}{2}} v_{\left.k+\frac{1}{2}, \ell-\frac{1}{2}\right\}}\right\} \text { for } u, v \in \mathcal{E}_{\mathrm{per}}^{2},} \\
& {[u \| v]_{3}=\frac{\sqrt{3} h^{2}}{4} \sum_{k=1}^{n} \sum_{\ell=1}^{n}\left\{u_{k, \ell-\frac{1}{2}} v_{k, \ell-\frac{1}{2}}+u_{k, \ell+\frac{1}{2}} v_{k, \ell+\frac{1}{2}}\right\}, \text { for } u, v \in \mathcal{E}_{\mathrm{per}}^{3} \text {. }}
\end{aligned}
$$

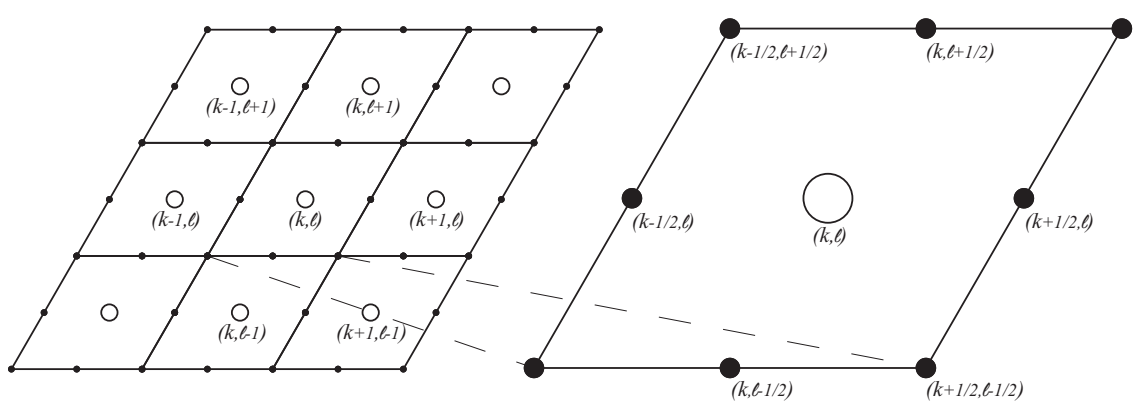

Figure 5: Numbering scheme for the cell-centered hexagonal grid. Logically, this numbering method is the same as for a square Cartesian grid, and, therefore, in our computations, we can use standard two-dimensional arrays to store the grid variables. The cell centered points are represented with open circles.

We define the center-to-edge differences:

$$
\begin{aligned}
D_{1} \phi_{k+\frac{1}{2}, \ell} & :=\frac{\phi_{k+1, \ell}-\phi_{k, \ell}}{h} \in \mathcal{E}_{\mathrm{per}}^{1} \\
D_{2} \phi_{k-\frac{1}{2}, \ell+\frac{1}{2}} & :=\frac{\phi_{k-1, \ell+1}-\phi_{k, \ell}}{h} \in \mathcal{E}_{\mathrm{per}}^{2} \\
D_{3} \phi_{k, \ell-\frac{1}{2}} & :=\frac{\phi_{k, \ell-1}-\phi_{k, \ell}}{h} \in \mathcal{E}_{\mathrm{per}}^{3}
\end{aligned}
$$


for $\phi \in \mathcal{C}_{\text {per. }}$ In a similar way, we define the edge-to-center differences as follows:

$$
\begin{aligned}
d_{1} u_{k, \ell} & :=\frac{u_{k+\frac{1}{2}, \ell}-u_{k-\frac{1}{2}, \ell}}{h} \in \mathcal{C}_{\mathrm{per}}, \quad \text { for } u \in \mathcal{E}_{\mathrm{per}}^{1} \\
d_{2} u_{k, \ell} & :=\frac{u_{k-\frac{1}{2}, \ell+\frac{1}{2}}-u_{k+\frac{1}{2}, \ell-\frac{1}{2}}}{h} \in \mathcal{C}_{\mathrm{per}}, \quad \text { for } u \in \mathcal{E}_{\mathrm{per}}^{2}, \\
d_{3} u_{k, \ell} & :=\frac{u_{k, \ell-\frac{1}{2}}-u_{k, \ell+\frac{1}{2}}}{h} \in \mathcal{C}_{\mathrm{per}}, \quad \text { for } u \in \mathcal{E}_{\Omega}^{3} .
\end{aligned}
$$

The edge-to-center averages are defined similarly:

$$
\begin{aligned}
& a_{1} u_{k, \ell}:=\frac{u_{k+\frac{1}{2}, \ell}+u_{k-\frac{1}{2}, \ell}}{2} \in \mathcal{C}_{\mathrm{per}}, \quad \text { for } u \in \mathcal{E}_{\mathrm{per}}^{1}, \\
& a_{2} u_{k, \ell}:=\frac{u_{k-\frac{1}{2}, \ell+\frac{1}{2}}+u_{k+\frac{1}{2}, \ell-\frac{1}{2}}}{2} \in \mathcal{C}_{\mathrm{per}}, \quad \text { for } u \in \mathcal{E}_{\mathrm{per}}^{2}, \\
& a_{3} u_{k, \ell}:=\frac{u_{k, \ell-\frac{1}{2}}+u_{k, \ell+\frac{1}{2}}}{2} \in \mathcal{C}_{\mathrm{per}}, \quad \text { for } u \in \mathcal{E}_{\mathrm{per}}^{3} .
\end{aligned}
$$

The discrete Laplacian is defined as follows: for $u \in \mathcal{C}_{\text {per }}$

$$
\Delta_{h} u_{k, \ell}:=\frac{2}{3}\left[d_{1}\left(D_{1} u\right)_{k, \ell}+d_{2}\left(D_{2} u\right)_{k, \ell}+d_{3}\left(D_{3} u\right)_{k, \ell}\right] \in \mathcal{C}_{\mathrm{per}}
$$

We need the following summation-by-parts results, whose proofs are omitted for the sake of brevity. We note that the periodicity of the grid functions are utilized in a fundamental way in the proofs.

Theorem 3.4. Suppose that $\phi, \psi \in \mathcal{C}_{\text {per }}(\Omega)$. Then

$$
\begin{aligned}
\left(d_{j}\left(D_{j} \phi\right) \| \psi\right) & =-\left[D_{j} \phi \| D_{j} \psi\right]_{j}, \quad j=1,2,3 \\
\left(a_{j} D_{j}(\phi) \| \psi\right) & =-\left(\phi \| a_{j} D_{j}(\psi)\right), \quad j=1,2,3 \\
\left(\Delta_{h} \phi \| \psi\right) & =\left(\phi \| \Delta_{h} \psi\right) .
\end{aligned}
$$

We now have all the tools necessary to introduce the discrete gradient energies: for any system of grid functions $\boldsymbol{u}, \boldsymbol{v} \in\left[\mathcal{C}_{\text {per }}(\Omega)\right]^{3}$, define

$$
E_{u, h}^{\mathcal{G}}(\boldsymbol{u}, \boldsymbol{v}):=\frac{1}{2} \sum_{j=1}^{3}\left[\left(\Delta_{h} u_{j} \| \Delta_{h} u_{j}\right)-4\left(a_{j}\left(D_{j} v_{j}\right) \| \Delta_{h} u_{j}\right)+4\left[D_{j} v_{j} \| D_{j} v_{j}\right]_{j}\right]
$$

and

$$
E_{v, h}^{\mathcal{G}}(\boldsymbol{u}, \boldsymbol{v}):=\frac{1}{2} \sum_{j=1}^{3}\left[\left(\Delta_{h} v_{j} \| \Delta_{h} v_{j}\right)+4\left(a_{j}\left(D_{j} u_{j}\right) \| \Delta_{h} v_{j}\right)+4\left[D_{j} u_{j} \| D_{j} u_{j}\right]_{j}\right] .
$$

The following properties are important to our analysis.

Proposition 3.5. The energies $E_{u, h}^{\mathcal{G}}(\boldsymbol{u}, \boldsymbol{v}), E_{v, h}^{\mathcal{G}}(\boldsymbol{u}, \boldsymbol{v})$ are consistent with their continuous-space counterparts defined in (2.34) to second order in $h$, as $h \rightarrow 0$. Moreover, for any system of grid functions $\boldsymbol{u}, \boldsymbol{v} \in\left[\mathcal{C}_{\text {per }}(\Omega)\right]^{3}$, we have

$$
E_{u, h}^{\mathcal{G}}(\boldsymbol{u}, \boldsymbol{v}) \geq 0, \quad E_{v, h}^{\mathcal{G}}(\boldsymbol{u}, \boldsymbol{v}) \geq 0
$$


Proof. The first part can be established by Taylor expansions, or even Fourier analysis methods. We suppress the details. For the second part, observe, for example, that

$$
\begin{aligned}
& \left(\Delta_{h} u_{1} \| \Delta_{h} u_{1}\right)-4\left(a_{1}\left(D_{1} v_{1}\right) \| \Delta_{h} u_{1}\right)+4\left[D_{1} v_{1} \| D_{1} v_{1}\right]_{1} \\
& =\frac{\sqrt{3} h^{2}}{2} \sum_{k=1}^{n} \sum_{\ell=1}^{n}\left(\Delta_{h}\left(u_{1}\right)_{k, \ell}-2 a_{1} D_{1}\left(v_{1}\right)_{k, \ell}\right)^{2} \geq 0
\end{aligned}
$$

Similar estimates hold for the other terms, and the result is proved.

We have the following discrete analog of Proposition 2.7 .

Proposition 3.6. Suppose that $\boldsymbol{u}, \boldsymbol{v} \in\left[\mathcal{C}_{\mathrm{per}}(\Omega)\right]^{3}$ are periodic grid functions. The discrete gradient energy, defined via

$$
E_{h}^{\mathcal{G}}(\boldsymbol{u}, \boldsymbol{v}):=E_{u, h}^{\mathcal{G}}(\boldsymbol{u}, \boldsymbol{v})+E_{v, h}^{\mathcal{G}}(\boldsymbol{u}, \boldsymbol{v}),
$$

is non-negative and convex, and the discrete variations are given by

$$
\delta_{u_{j}} E_{h}^{\mathcal{G}}=\Delta_{h}^{2} u_{j}-4 a_{j} D_{j}\left(\Delta_{h} v_{j}\right)-4 d_{j}\left(D_{j} u_{j}\right)
$$

and

$$
\delta_{v_{j}} E_{h}^{\mathcal{G}}=\Delta_{h}^{2} v_{j}+4 a_{j} D_{j}\left(\Delta_{h} u_{j}\right)-4 d_{j}\left(D_{j} v_{j}\right)
$$

Proof. The non-negativity follows by simple rearrangements of terms, as indicated above. To prove the other results, we need to calculate the discrete variations. To this end, let $\phi, \psi \in\left[\mathcal{C}_{\text {per }}(\Omega)\right]^{3}$ be periodic grid functions. Then, as in the continuous case, the following identities are available:

$$
\begin{aligned}
d_{s} E_{u, h}^{\mathcal{G}} & (\boldsymbol{u}+s \boldsymbol{\phi}, \boldsymbol{v}+s \boldsymbol{\psi}) \\
= & \sum_{j=1}^{3}\left(\Delta_{h}\left(u_{j}+s \phi_{j}\right) \| \Delta_{h} \phi_{j}\right)+\sum_{j=1}^{3} 4\left[D_{j}\left(v_{j}+s \psi_{j}\right) \| D_{j} \psi_{j}\right]_{j} \\
& \quad-\frac{1}{2} \sum_{j=1}^{3}\left\{4\left(a_{j}\left(D_{j} \psi_{j}\right) \| \Delta_{h}\left(u_{j}+s \phi_{j}\right)\right)+4\left(a_{j}\left(D_{j}\left(v_{j}+s \psi_{j}\right)\right) \| \Delta_{h} \phi_{j}\right)\right\},
\end{aligned}
$$

and

$$
\begin{aligned}
d_{s} E_{v, h}^{\mathcal{G}}(\boldsymbol{u}+s \boldsymbol{\phi}, \boldsymbol{v}+s \boldsymbol{\psi}) & \\
= & \sum_{j=1}^{3}\left(\Delta_{h}\left(v_{j}+s \psi_{j}\right) \| \Delta_{h} \psi_{j}\right)+\sum_{j=1}^{3} 4\left[D_{j}\left(u_{j}+s \phi_{j}\right) \| D_{j} \phi_{j}\right]_{j} \\
& +\frac{1}{2} \sum_{j=1}^{3}\left\{4\left(a_{j}\left(D_{j} \phi_{j}\right) \| \Delta_{h}\left(v_{j}+s \psi_{j}\right)\right)+4\left(a_{j}\left(D_{j}\left(u_{j}+s \phi_{j}\right)\right) \| \Delta_{h} \psi_{j}\right)\right\} .
\end{aligned}
$$

Using the discrete periodicity of the fields with the appropriate summation-by-parts formulas and the fact that the operators $a_{j}\left(D_{j}\right)$ and $\Delta_{h}$ commute, we find that

$$
\begin{aligned}
\left.d_{s} E_{u, h}^{\mathcal{G}}(\boldsymbol{u}+s \boldsymbol{\phi}, \boldsymbol{v}+s \boldsymbol{\psi})\right|_{s=0}= & \sum_{j=1}^{3}\left(\Delta_{h}^{2} u_{j} \| \phi_{j}\right)-\sum_{j=1}^{3} 4\left(d_{j}\left(D_{j} v_{j}\right) \| \psi_{j}\right) \\
& +\sum_{j=1}^{3}\left\{2\left(a_{j} D_{j}\left(\Delta_{h} u_{j}\right) \| \psi_{j}\right)-2\left(a_{j} D_{j}\left(\Delta_{h} v_{j}\right) \| \phi_{j}\right)\right\},
\end{aligned}
$$


and

$$
\begin{aligned}
\left.d_{s} E_{v, h}^{\mathcal{G}}(\boldsymbol{u}+s \boldsymbol{\phi}, \boldsymbol{v}+s \boldsymbol{\psi})\right|_{s=0}= & \sum_{j=1}^{3}\left(\Delta_{h}^{2} v_{j} \| \psi_{j}\right)-\sum_{j=1}^{3} 4\left(d_{j}\left(D_{j} u_{j}\right) \| \phi_{j}\right) \\
& +\sum_{j=1}^{3}\left\{-2\left(a_{j} D_{j}\left(\Delta_{h} v_{j}\right) \| \phi_{j}\right)+2\left(a_{j} D_{j}\left(\Delta_{h} u_{j}\right) \| \psi_{j}\right)\right\},
\end{aligned}
$$

from which we get the discrete variational derivatives in (3.41) and (3.42).

The convexity of the energy follows upon calculating the second variations, which are given by

$$
\begin{aligned}
d_{s}^{2} E_{u, h}^{\mathcal{G}}(\boldsymbol{u}+s \boldsymbol{\phi}, \boldsymbol{v}+s \boldsymbol{\psi})= & \sum_{j=1}^{3}\left(\Delta_{h} \phi_{j} \| \Delta_{h} \phi_{j}\right)+\sum_{j=1}^{3} 4\left[D_{j} \psi_{j} \| D_{j} \psi_{j}\right]_{j} \\
& -\frac{1}{2} \sum_{j=1}^{3}\left\{4\left(a_{j}\left(D_{j} \psi_{j}\right) \| \Delta_{h} \phi_{j}\right)+4\left(a_{j}\left(D_{j} \psi_{j}\right) \| \Delta_{h} \phi_{j}\right)\right\} \\
= & E_{u, h}^{\mathcal{G}}(\boldsymbol{\phi}, \boldsymbol{\psi}) \geq 0,
\end{aligned}
$$

and

$$
\begin{aligned}
d_{s}^{2} E_{v, h}^{\mathcal{G}}(\boldsymbol{u}+s \boldsymbol{\phi}, \boldsymbol{v}+s \boldsymbol{\psi})= & \sum_{j=1}^{3}\left(\Delta_{h} \psi_{j} \| \Delta_{h} \psi_{j}\right)+\sum_{j=1}^{3} 4\left[D_{j} \phi_{j} \| D_{j} \phi_{j}\right]_{j} \\
& +\frac{1}{2} \sum_{j=1}^{3}\left\{4\left(a_{j}\left(D_{j} \phi_{j}\right) \| \Delta_{h} \psi_{j}\right)+4\left(a_{j}\left(D_{j} \phi_{j}\right) \| \Delta_{h} \psi_{j}\right)\right\} \geq 0 \\
= & E_{v, h}^{\mathcal{G}}(\boldsymbol{\phi}, \boldsymbol{\psi}) \geq 0 .
\end{aligned}
$$

Using the last result and Lemma 2.8 again, we have the following.

Theorem 3.7. Let $\boldsymbol{u}, \boldsymbol{v} \in\left[\mathcal{C}_{\text {per }}(\Omega)\right]^{3}$. Define the discrete energies

$$
E_{h, c}(\boldsymbol{u}, \boldsymbol{v})=\left(T_{c}(\boldsymbol{u}, \boldsymbol{v}) \| \mathbf{1}\right)+B_{4} E_{u, h}^{\mathcal{G}}(\boldsymbol{u}, \boldsymbol{v})+B_{4} E_{v, h}^{\mathcal{G}}(\boldsymbol{u}, \boldsymbol{v}), \quad E_{h, e}(\boldsymbol{u}, \boldsymbol{v})=\left(T_{e}(\boldsymbol{u}, \boldsymbol{v}) \| \mathbf{1}\right) .
$$

Then, $E_{h, c}$ and $E_{h, e}$ are convex, and the respective discrete variations are given by

$$
\begin{aligned}
\delta_{u_{j}} E_{h, c}= & B_{4}\left(\Delta_{h}^{2} u_{j}-4 a_{j} D_{j}\left(\Delta_{h} v_{j}\right)-4 d_{j}\left(D_{j} u_{j}\right)\right) \\
& +2 \max \left\{\frac{\left(6 \nu \rho_{0}-2 \tau\right)^{2}}{3 \nu},\left(\frac{B_{0}}{2}+\frac{3 \nu \rho_{0}^{2}}{2}-\tau \rho_{0}\right)\right\} u_{j} \\
& +6 \nu A^{2} u_{j}+\left(6 \nu \rho_{0}-2 \tau\right)\left(u_{\alpha_{j}} u_{\beta_{j}}-v_{\alpha_{j}} v_{\beta_{j}}\right), \\
\delta_{u_{j}} E_{h, e}= & 3 \nu\left(u_{j}^{2}+v_{j}^{2}\right) u_{j}+2 \max \left\{0, \frac{\left(6 \nu \rho_{0}-2 \tau\right)^{2}}{3 \nu}-\left(\frac{B_{0}}{2}+\frac{3 \nu \rho_{0}^{2}}{2}-\tau \rho_{0}\right)\right\} u_{j},
\end{aligned}
$$

and

$$
\begin{aligned}
\delta_{v_{j}} E_{h, c}= & B_{4}\left(\Delta_{h}^{2} v_{j}+4 a_{j} D_{j}\left(\Delta_{h} u_{j}\right)-4 d_{j}\left(D_{j} v_{j}\right)\right) \\
& +2 \max \left\{\frac{\left(6 \nu \rho_{0}-2 \tau\right)^{2}}{3 \nu},\left(\frac{B_{0}}{2}+\frac{3 \nu \rho_{0}^{2}}{2}-\tau \rho_{0}\right)\right\} v_{j}, \\
& +6 \nu A^{2} v_{j}-\left(6 \nu \rho_{0}-2 \tau\right)\left(u_{\alpha_{j}} v_{\beta_{j}}+v_{\alpha_{j}} u_{\beta_{j}}\right) \\
\delta_{v_{j} E_{h, e}=} & 3 \nu\left(u_{j}^{2}+v_{j}^{2}\right) v_{j}+2 \max \left\{0, \frac{\left(6 \nu \rho_{0}-2 \tau\right)^{2}}{3 \nu}-\left(\frac{B_{0}}{2}+\frac{3 \nu \rho_{0}^{2}}{2}-\tau \rho_{0}\right)\right\} v_{j} .
\end{aligned}
$$


We formulate the following (first-order in time) fully discrete, energy stable convex splitting scheme. Suppose $s>0$ is the time step size and that $\boldsymbol{u}_{0}, \boldsymbol{v}_{0} \in\left[C_{\text {per }}^{\infty}(\mathrm{D})\right]^{3}$. Set $\boldsymbol{u}^{0}=\mathcal{P}_{h}\left(\boldsymbol{u}_{0}\right) \in\left[\mathcal{C}_{\text {per }}\right]^{3}$ and $\boldsymbol{v}^{0}=\mathcal{P}_{h}\left(\boldsymbol{v}_{0}\right) \in\left[\mathcal{C}_{\text {per }}\right]^{3}$, where $\mathcal{P}_{h}$ is the standard point-wise projection of a continuous function into $\mathcal{C}_{\text {per }}$. Then, for $j=1,2,3$, and for $m=0, \ldots, M-1$, where $s M=T$, the scheme is given by

$$
\begin{aligned}
u_{j}^{m+1}-u_{j}^{m}= & -s \mu_{j}^{m+1}, \\
\mu_{j}^{m+1}= & B_{4}\left(\Delta_{h}^{2} u_{j}^{m+1}-4 a_{j} D_{j}\left(\Delta_{h} v_{j}^{m+1}\right)-4 d_{j}\left(D_{j} u_{j}^{m+1}\right)\right) \\
& +2 \max \left\{\frac{\left(6 \nu \rho_{0}-2 \tau\right)^{2}}{3 \nu},\left(\frac{B_{0}}{2}+\frac{3 \nu \rho_{0}^{2}}{2}-\tau \rho_{0}\right)\right\} u_{j}^{m+1} \\
& +6 \nu\left(A^{m+1}\right)^{2} u_{j}^{m+1}-3 \nu\left(\left(u_{j}^{m}\right)^{2}+\left(v_{j}^{m}\right)^{2}\right) u_{j}^{m} \\
& +\left(6 \nu \rho_{0}-2 \tau\right)\left(u_{\alpha_{j}}^{m+1} u_{\beta_{j}}^{m+1}-v_{\alpha_{j}}^{m+1} v_{\beta_{j}}^{m+1}\right) \\
& -2 \max \left\{0, \frac{\left(6 \nu \rho_{0}-2 \tau\right)^{2}}{3 \nu}-\left(\frac{B_{0}}{2}+\frac{3 \nu \rho_{0}^{2}}{2}-\tau \rho_{0}\right)\right\} u_{j}^{m}, \\
v_{j}^{m+1}-v_{j}^{m}= & -s \nu_{j}^{m+1}, \\
\nu_{j}^{m+1}= & B_{4}\left(\Delta_{h}^{2} v_{j}^{m+1}+4 a_{j} D_{j}\left(\Delta_{h} u_{j}^{m+1}\right)-4 d_{j}\left(D_{j} v_{j}^{m+1}\right)\right) \\
& +2 \max \left\{\frac{\left(6 \nu \rho_{0}-2 \tau\right)^{2}}{3 \nu},\left(\frac{B_{0}}{2}+\frac{3 \nu \rho_{0}^{2}}{2}-\tau \rho_{0}\right)\right\} v_{j}^{m+1} \\
& +6 \nu\left(A^{m+1}\right)^{2} v_{j}^{m+1}-3 \nu\left(\left(u_{j}^{m}\right)^{2}+\left(v_{j}^{m}\right)^{2}\right) v_{j}^{m} \\
& -\left(6 \nu \rho_{0}-2 \tau\right)\left(u_{\alpha_{j}}^{m+1} v_{\beta_{j}}^{m+1}+v_{\alpha_{j}}^{m+1} u_{\beta_{j}}^{m+1}\right) \\
& -2 \max \left\{0, \frac{\left(6 \nu \rho_{0}-2 \tau\right)^{2}}{3 \nu}-\left(\frac{B_{0}}{2}+\frac{3 \nu \rho_{0}^{2}}{2}-\tau \rho_{0}\right)\right\} v_{j}^{m} .
\end{aligned}
$$

Because the scheme respects the convexity of the energy, we have the following solvability result $[41,45]$.

Corollary 3.8. Let $\boldsymbol{u}^{m}, \boldsymbol{v}^{m} \in\left[\mathcal{C}_{\mathrm{per}}\right]^{3}$. The fully discrete scheme (3.54) - (3.57) is uniquely solvable for any step size $s>0$.

Proof. This follows from the strict convexity and coercivity of the functional

$$
\begin{aligned}
G(\boldsymbol{u}, \boldsymbol{v})= & \frac{1}{2 s} \sum_{j=1}^{3}\left(\left\|u_{j}-u_{j}^{m}\right\|_{2}^{2}+\left\|v_{j}-v_{j}^{m}\right\|_{2}^{2}\right)+E_{h, c}(\boldsymbol{u}, \boldsymbol{v}) \\
& -\sum_{j=1}^{3}\left(\delta_{u_{j}} E_{h, e}\left(\boldsymbol{u}^{m}, \boldsymbol{v}^{m}\right) \| u_{j}\right)-\sum_{j=1}^{3}\left(\delta_{v_{j}} E_{h, e}\left(\boldsymbol{u}^{m}, \boldsymbol{v}^{m}\right) \| v_{j}\right) .
\end{aligned}
$$

We address the coercivity of the whole discrete energy $E_{h}$ below in Theorem 3.11.

We now state the discrete analog of Theorem 3.1, whose proof is omitted for brevity.

Theorem 3.9. Suppose that $\boldsymbol{\phi}, \boldsymbol{\psi} \in\left[\mathcal{C}_{\mathrm{per}}\right]^{6}$. Consider a convex splitting of a discrete energy $E_{h}(\boldsymbol{u}, \boldsymbol{v}):=E_{h, c}(\boldsymbol{u}, \boldsymbol{v})-E_{h, e}(\boldsymbol{u}, \boldsymbol{v})$ of the type given in Theorem 3.7. Then

$$
E_{h}(\boldsymbol{\phi})-E_{h}(\boldsymbol{\psi}) \leq\left(\boldsymbol{\delta} E_{h, c}(\boldsymbol{\phi})-\boldsymbol{\delta} E_{h, e}(\boldsymbol{\psi}) \| \boldsymbol{\phi}-\boldsymbol{\psi}\right),
$$

where $\boldsymbol{\delta}$ now denotes the discrete variational derivative. 
Corollary 3.10. Set $E_{h}(\boldsymbol{u}, \boldsymbol{v}):=E_{h, c}(\boldsymbol{u}, \boldsymbol{v})-E_{h, e}(\boldsymbol{u}, \boldsymbol{v})$, with $E_{h, c}, E_{h, e}$ as in Theorem 3.7. Let $\boldsymbol{u}^{m+1}, \boldsymbol{u}^{m}, \boldsymbol{v}^{m+1}, \boldsymbol{v}^{m} \in\left[\mathcal{C}_{\mathrm{per}}\right]^{3}$ be the unique solutions of the fully discrete scheme $(3.54)-(3.57)$. Then

$$
E_{h}\left(\boldsymbol{u}^{m+1}, \boldsymbol{v}^{m+1}\right)+s \sum_{j=1}^{3}\left(\mu_{j}^{m+1} \| \mu_{j}^{m+1}\right)+s \sum_{j=1}^{3}\left(\nu_{j}^{m+1} \| \nu_{j}^{m+1}\right) \leq E_{h}\left(\boldsymbol{u}^{m}, \boldsymbol{v}^{m}\right) .
$$

The proof of the following discrete analog of Theorem 2.10 is similar to that of the continuous version and is omitted for brevity.

Theorem 3.11. For the discrete energy $E_{h}(\boldsymbol{u}, \boldsymbol{v}):=E_{h, c}(\boldsymbol{u}, \boldsymbol{v})-E_{h, e}(\boldsymbol{u}, \boldsymbol{v})$, with $E_{h, c}, E_{h, e}$ as in Theorem 3.7, we have the following lower bound

$$
E_{h}(\boldsymbol{u}, \boldsymbol{v}) \geq \gamma_{1} \sum_{j=1}^{3}\left(\left\|\Delta_{h} u_{j}\right\|_{2}^{2}+\left\|\Delta_{h} v_{j}\right\|_{2}^{2}+\left\|u_{j}\right\|_{2}^{2}+\left\|v_{j}\right\|_{2}^{2}\right)-\chi_{1},
$$

for any $\boldsymbol{u}, \boldsymbol{v} \in\left[\mathcal{C}_{\mathrm{per}}\right]^{3}$, for some positive constants $\gamma_{1}$ and $\chi_{1}$ that depend on $B, \nu, \tau, B_{4}$ and $\mathrm{D}$ but are independent of $h>0$.

A combination of Corollary 3.10 and Theorem 3.11 implies the following result:

Corollary 3.12. Set $E_{h}(\boldsymbol{u}, \boldsymbol{v}):=E_{h, c}(\boldsymbol{u}, \boldsymbol{v})-E_{h, e}(\boldsymbol{u}, \boldsymbol{v})$, with $E_{h, c}, E_{h, e}$ as in Theorem 3.7. Suppose that $\boldsymbol{u}_{0}, \boldsymbol{v}_{0} \in\left[C_{\mathrm{per}}^{\infty}(\mathrm{D})\right]^{3}$. Let $\boldsymbol{u}^{m}, \boldsymbol{v}^{m} \in\left[\mathcal{C}_{\mathrm{per}}\right]^{3}, m=0, \ldots, M$, be solutions of the fully scheme (3.54) - (3.57), where $\boldsymbol{u}^{0}=\mathcal{P}_{h}\left(\boldsymbol{u}_{0}\right)$ and $\boldsymbol{v}^{0}=\mathcal{P}_{h}\left(\boldsymbol{v}_{0}\right)$. Then

$$
\begin{aligned}
\max _{0 \leq m \leq M}\left[\gamma _ { 1 } \sum _ { j = 1 } ^ { 3 } \left(\left\|\Delta_{h} u_{j}^{m}\right\|_{2}^{2}+\left\|\Delta_{h} v_{j}^{m}\right\|_{2}^{2}+\right.\right. & \left.\left.\left\|u_{j}^{m}\right\|_{2}^{2}+\left\|v_{j}^{m}\right\|_{2}^{2}\right)\right] \\
& +s \sum_{m=1}^{M}\left[\sum_{j=1}^{3}\left\|\mu_{j}^{m}\right\|_{2}^{2}+\sum_{j=1}^{3}\left\|\nu_{j}^{m}\right\|_{2}^{2}\right] \leq C_{5},
\end{aligned}
$$

where $C_{5}>0$ is independent of $h>0$ and $s>0$.

In analogy with the previous results on a square mesh [45], we have the following discrete Sobolev inequality:

Theorem 3.13. For any $u \in \mathcal{C}_{\text {per }}$, there is a constant $C_{6}>0$ independent of $h>0$, such that

$$
\|u\|_{\infty} \leq C_{6} \sqrt{\left\|\Delta_{h} u\right\|_{2}^{2}+\|u\|_{2}^{2}}
$$

Then it follows that

Corollary 3.14. With the same assumptions as in Corollary 3.12, we have the point-wise stability

$$
\max _{\substack{0 \leq m \leq M \\ 1 \leq j \leq 3}}\left[\left\|u_{j}^{m}\right\|_{\infty}+\left\|v_{j}^{m}\right\|_{\infty}\right] \leq C_{7}
$$

where $C_{7}>0$ is independent of $h>0$ and $s>0$.

Using the last stability, together with the consistency of the scheme, we can rigorously prove error estimates, with optimal rates of convergence, as in [45]. For the sake of brevity, we skip the error estimates herein. 


\section{Numerical Results}

In this section we present the results of three numerical experiments demonstrating the convergence and stability of the proposed schemes.

\subsection{Numerical Convergence Test}

The first experiment verifies the convergence rate with the following setting: (i) the computation domain $\mathrm{D}$ is the scaled reciprocal cell with the scalar factor $\sigma=1$; (ii) the initial data are expressed as

$$
u_{j}(\boldsymbol{x}, 0)=\cos \left(\boldsymbol{a}_{1} \cdot \boldsymbol{x}\right)+\cos \left(\boldsymbol{a}_{2} \cdot \boldsymbol{x}\right), \quad v_{j}(\boldsymbol{x}, 0)=\sin \left(\boldsymbol{a}_{1} \cdot \boldsymbol{x}\right)+\sin \left(\boldsymbol{a}_{2} \cdot \boldsymbol{x}\right),
$$

for $j=1,2,3$, with Bravais lattice vector $\boldsymbol{a}_{1}$ and $\boldsymbol{a}_{2}$ defined as in (2.3); (iii) $\nu=1, \tau=0$, $\rho_{0}=-0.195, B=-0.128150, B_{4}=1.0$; (iv) the time step $s$ and the grid size $h$ are related by the quadratic refinement path $s=0.1 h^{2} ;(\mathrm{v})$ in all cases, the final time is

$$
T=10 \cdot \frac{0.1}{32^{2}}=40 \cdot \frac{0.1}{64^{2}}=160 \cdot \frac{0.1}{128^{2}}=640 \cdot \frac{0.1}{256^{2}}=2560 \cdot \frac{0.1}{512^{2}} .
$$

The initial conditions and the results of simulation are presented in Figure 6 for the case $h=1 / 128$.

Table 1: Numerical Convergence Test using a quadratic refinement path. The parameters for the test are given in the text. The results of the test suggest that the scheme is second order accurate in space and first order in time, as predicted.

\begin{tabular}{|c|c|c|c|}
\hline coarse,$h_{c}$ & fine, $h_{f}$ & $\delta$ & rate \\
\hline $1 / 32$ & $1 / 64$ & 0.009271065152390 & - \\
\hline $1 / 64$ & $1 / 128$ & 0.002068816022822 & 2.163929745695177 \\
\hline $1 / 128$ & $1 / 256$ & 0.000500230455258 & 2.048140553745060 \\
\hline $1 / 256$ & $1 / 512$ & 0.000123975086732 & 2.012542660355964 \\
\hline
\end{tabular}

Since there is no exact solution with which to compare, we use the difference between results on relative coarse and fine grids to quantify convergence, as in [25]. We denote $\delta u_{j}:=u_{j}^{h_{f}}-u_{j}^{h_{c}}$, where $h_{f}$ is the fine grid spacing and $h_{c}$ is the coarse grid spacing. We then set

$$
\delta:=\frac{1}{6} \sum_{j=1}^{3}\left[\left\|\delta u_{j}\right\|_{2}+\left\|\delta v_{j}\right\|_{2}\right] .
$$

We expect, since we use a quadratic refinement path that numerical "error" will satisfy, as $h \rightarrow 0$,

$$
\delta=\mathcal{O}\left(h^{2}\right)+\mathcal{O}(s)=\mathcal{O}\left(h^{2}\right)+\mathcal{O}\left(0.1 h^{2}\right)=\mathcal{O}\left(h^{2}\right) .
$$

The results are given in Table 1, and the convergence order obtained from the test provides evidence that the scheme is indeed second order accurate in space and first order in time.

Remark 4.1. Note that we use the quadratic refinement path not for the purposes of stability indeed the algorithm is unconditionally stable - but solely to achieve the quadratic convergence in our test. We are currently developing a second-order (in time) numerical scheme for this problem, and, with that in hand, we could use a linear refinement path to achieve quadratic convergence. 

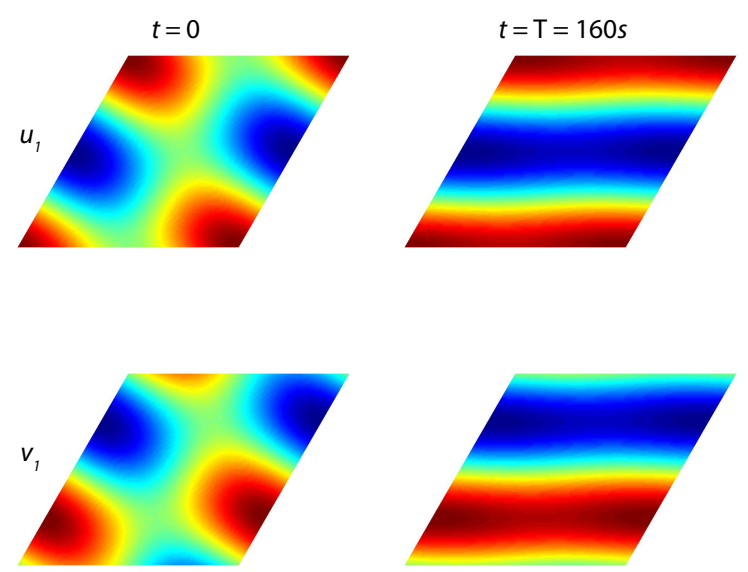

Figure 6: The initial data and final values of $u_{1}$ and $v_{1}$ in the convergence test. Here $h=1 / 128$.

\subsection{Grain Rotation and Shrinkage}

In this section we simulate the rotation and shrinkage of an embedded circular grain in an otherwise perfect two-dimensional crystal. In particular, a circular grain in the center of a periodic domain is initially rotated by a certain angle creating dislocations at the boundary between the grain and the surrounding crystal. We explore the dynamics of the system, confirming energy dissipation, for instance, and compare results obtained with different sizes of time step $s$.
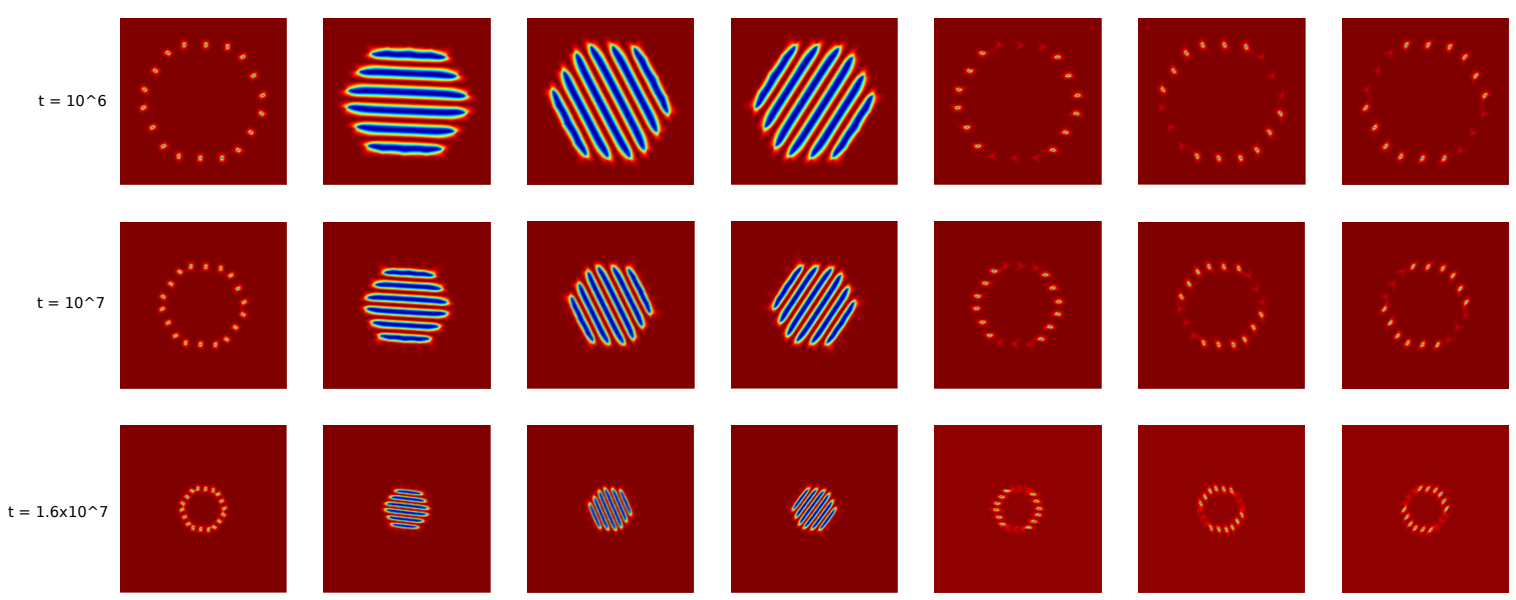

Figure 7: The rotation and shrinkage of a circular grain in an otherwise perfect crystal. Here we have zoomed in on that portion of the hexagonal computational domain showing the embedded, circular grain. Time increases from top to bottom. In columns the following are plotted: (column 1) $\sum_{j=1}^{3}\left|\eta_{j}\right| ;($ columns $2-4) u_{j}, j=1,2,3 ;($ columns $5-$ 7) $\left|\eta_{j}\right|, j=1,2,3$. The grain rotates slightly as its radius shrinks.

The first numerical experiment uses the following set-up: (i) the computation domain $\mathrm{D}$ is the scaled reciprocal cell, with the scaling factor $\sigma=1024$; (ii) $h=\frac{1024}{256}=4 ; s=100$; (iii) $\nu=1$, $\tau=0, \rho_{0}=-0.195, B_{0}=-0.128150, B_{4}=1$; (iv) the initial condition is a circular rotated grain 

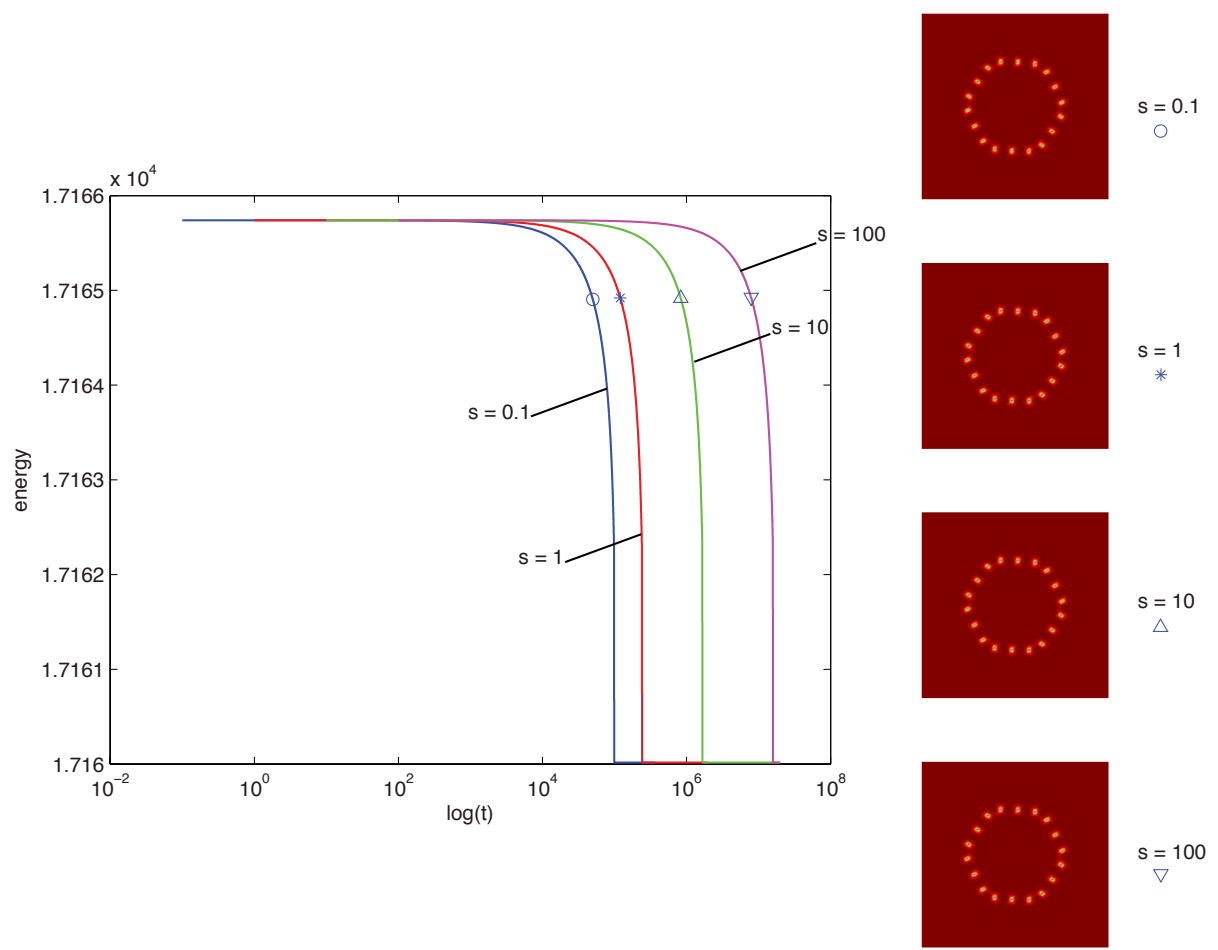

Figure 8: Energy dissipation for the embedded circular grain simulation, using four different time step sizes. The parameters are contained in the text. In particular, $h=\frac{1024}{256}=4$ is held fixed. The plots in the column on the right show the respective outputs for the different runs at roughly the same energy value. Note that the sizes of the grains are nearly indistinguishable as might be expected.

with radius 256 located in the center of D. Specifically, for $\boldsymbol{x}$ inside the rotated grain

$$
\begin{aligned}
& \boldsymbol{u}_{j}(\boldsymbol{x}, 0)=\alpha \cos \left(\boldsymbol{g}_{j} \cdot \mathrm{R}(\theta)(\boldsymbol{x}-\boldsymbol{c})^{T}\right), \\
& \boldsymbol{v}_{j}(\boldsymbol{x}, 0)=\alpha \sin \left(\boldsymbol{g}_{j} \cdot \mathrm{R}(\theta)(\boldsymbol{x}-\boldsymbol{c})^{T}\right),
\end{aligned}
$$

for $j=1,2,3$. Here $\boldsymbol{c}$ is the coordinate vector for the center of $\mathrm{D}, \mathrm{R}(\theta)$ is the rotation matrix

$$
\mathrm{R}(\theta)=\left[\begin{array}{rr}
\cos (\theta) & \sin (\theta) \\
-\sin (\theta) & \cos (\theta)
\end{array}\right]
$$

with $\theta=0.0872665$ (radians), and $\alpha=0.08859166596650425$. Outside the rotated grain, we take $u_{j}(\boldsymbol{x}, 0)=\alpha$ and $v_{j}(\boldsymbol{x}, 0)=0$, for $j=1,2,3$. Figure 7 shows the amplitudes at three different time instances. Time increases from top to bottom. In the columns of the figure the following are plotted: (column 1) $\sum_{j=1}^{3}\left|\eta_{j}\right| ;($ columns $2-4) u_{j}, j=1,2,3$; (columns $\left.5-7\right)\left|\eta_{j}\right|, j=1,2,3$. This simulation indicates that the PFC framework predicts that the grain rotates as it shrinks. This can be inferred by the shortening of the wavelengths characterizing the amplitudes. See for comparison, Figure 4.

As stated in Proposition 2.6, amplitude equations should dissipate energy (2.29). The discrete algorithm preserves such a property with respect to the discrete energy (3.49), as proved in Corollary 3.10, no matter how large is the time step size. On the other hand, larger time step sizes can lead to increased error. In this next test, we try to quantify the error, at least for the present simple case of grain rotation and shrinkage, and show simultaneously, that the algorithm remains stable for relatively large time step size variations. 

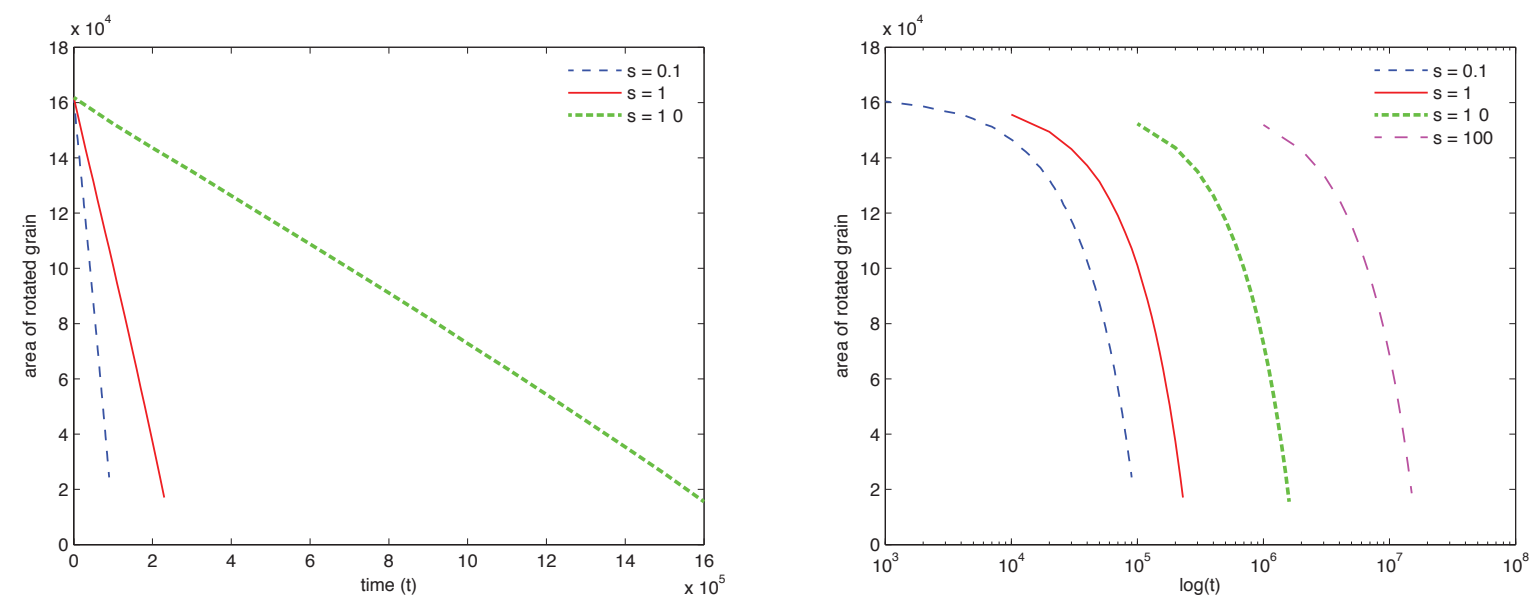

Figure 9: The evolution of the area inside the circular grain with different time step sizes, plotted as functions of time. The parameters are the same as in the simulations reported in Figure 8 . The plot on the right has the same information as that on the left, except that (i) the time is on the log scale on the right and (ii) we add the the data for the case $s=100$ on the right, since it fits better on a semi-log plot. From the plot on the left, a linear decrease in the area of the grain is observed for three different time step sizes.

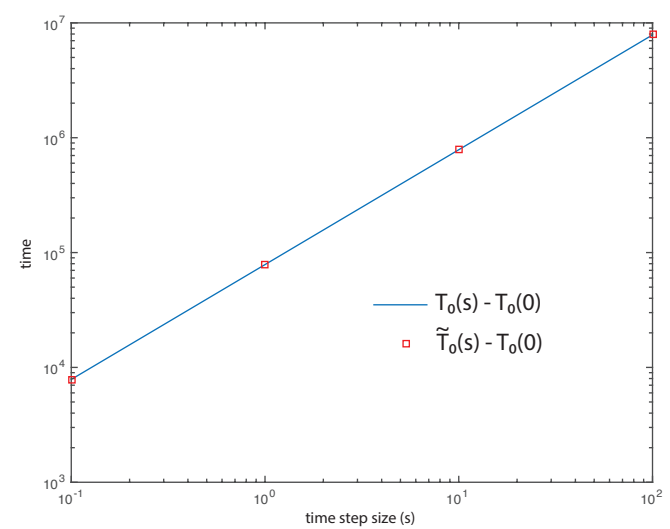

Figure 10: A plot of $T_{0}(s)$, the time at which the energy in the simulations featured in Figures 8 and 9 reaches the value $E_{0}=1.716492 \times 10^{4}$, as a function of the time step size $s$. $\tilde{T}(s)$ is the actual computed time to reach the energy $E_{0}$ for the four time step sizes $s=0.1,1.0,10.0$ and 100.0. Herein $T(s)$ is computed as the linear least squares best fit of the untransformed, computed data. The results agree with the linear approximation (4.3) remarkably well and give further evidence of the first-order convergence in time. In particular, our results suggest that the time to reach the energy $E_{0}$ for the exact PDE solution is precisely $T_{0}(0)=4.14096 \times 10^{4}$. 
The parameter setup for this next test is identical to the experiment shown in Figure 7, except that we use four different time step sizes, $s=0.1,1,10$ and 100. The spatial step size is held

fixed: $h=\frac{1024}{256}=4$. We also pick a specific time instant from each of the four evolutions, such that the energy is approximately the same. The numerical results of the amplitude equations are presented by plotting the energy curves with the four plots of $\sum_{j=1}^{3}\left|\eta_{j}\right|$ in Figure 8. The numerical energies are decreasing in all cases, which provides evidence of the unconditional stability of the schemes, though the dissipation rates are very different. Note that, if we compare the numerical results with different time step sizes and the same energy level, it can be observed that the profiles of amplitudes do not differ significantly. In particular, the number of dislocations, 18, is invariant.

The classical prediction of grain evolution states that the normal velocity of the grain boundary is proportional to its curvature. Thus, we also record the area of the rotated circular grain over time with different sizes of $s$. These results are illustrated in Figure 9. The paper by Heinonen, et al. [23] has reported a linear decrease in the area using a similar model, but a very different numerical method. The expected linear decrease in the area of the grain is observed in our simulations, though the constants of proportionality are quite different, and strongly depend on the time step size, $s$.

While the results of the test displayed in Figures 8 and 9 suggest that the accuracy of the simulations is severely degraded for large time step sizes, we can at least see that the results are consistent with a first-order in time approximation. Define $T_{0}(s)$ to be the time at which the energy in the simulation reaches the value $1.716492 \times 10^{4}$ for a given time step size $s$. Provided $h$ is small enough so that spatial errors can be neglected, and for sufficiently small $s$, we speculate that the following first-order approximation is valid:

$$
T_{0}(s)=T_{0}(0)+a \cdot s,
$$

for some positive constant $T_{0}(0)$ and some slope constant $a$. Our computational results yield the data

$$
\tilde{T}_{0}(0.1)=49,268, \quad \tilde{T}_{0}(1.0)=120,000, \quad \tilde{T}_{0}(10)=827320, \quad \tilde{T}_{0}(100)=7900500,
$$

the tilde indicating the imperfection in the computational values. These values can be gleaned from a careful analysis of the data used to construct Figure 8 . To construct $T(s)$, we use a linear least squares best fit of the four untransformed, computed data points $\tilde{T}(s)$, obtaining $T_{0}(0)=$ $4.14096 \times 10^{4}$ and $a=7.8591 \times 10^{4}$. In Figure 10 , we plot $\tilde{T}_{0}(s)-T_{0}(0)$ and $T_{0}(s)-T_{0}(0)$ versus $s$, and we find remarkable agreement, which gives further evidence that the scheme is first order accurate in time.

\subsection{Formation of Grain Boundaries}

Amplitude equation is also capable of simulating crystal grain growth. This numerical experiment uses the following setting: (i) The computational domain $\mathrm{D}$ is defined via scaling factor $\sigma=512$; (ii) $h=\frac{512}{256}=2, s=10$; (iii) $\nu=1, \tau=0, \rho_{0}=-0.25, B=-0.2, B_{4}=1$; (iv) the initial condition is defined similar to equation (4.1) in the grain rotation experiment. Here we have four small rotation regions located at four quadrants of the computational domain, with different rotation angles. These work as the "seeds" of four separate grains. Outside the four seeds, the amplitudes are zero, representing a kind of pseudo-liquid phase. The numerical results are presented in Figure 11. The first column shows the evolution of the reconstructed density field $\rho$, obtained using (2.7). Due to the different resolution required, the grid size of $\rho$ is much smaller than the amplitude equations. The second and third columns are plots of $\sum_{j=1}^{3}\left|\eta_{j}\right|$ and $\boldsymbol{u}_{1}$, respectively. In this experiment we observe the formation of grain boundary clearly. We also point out that in order to obtain 

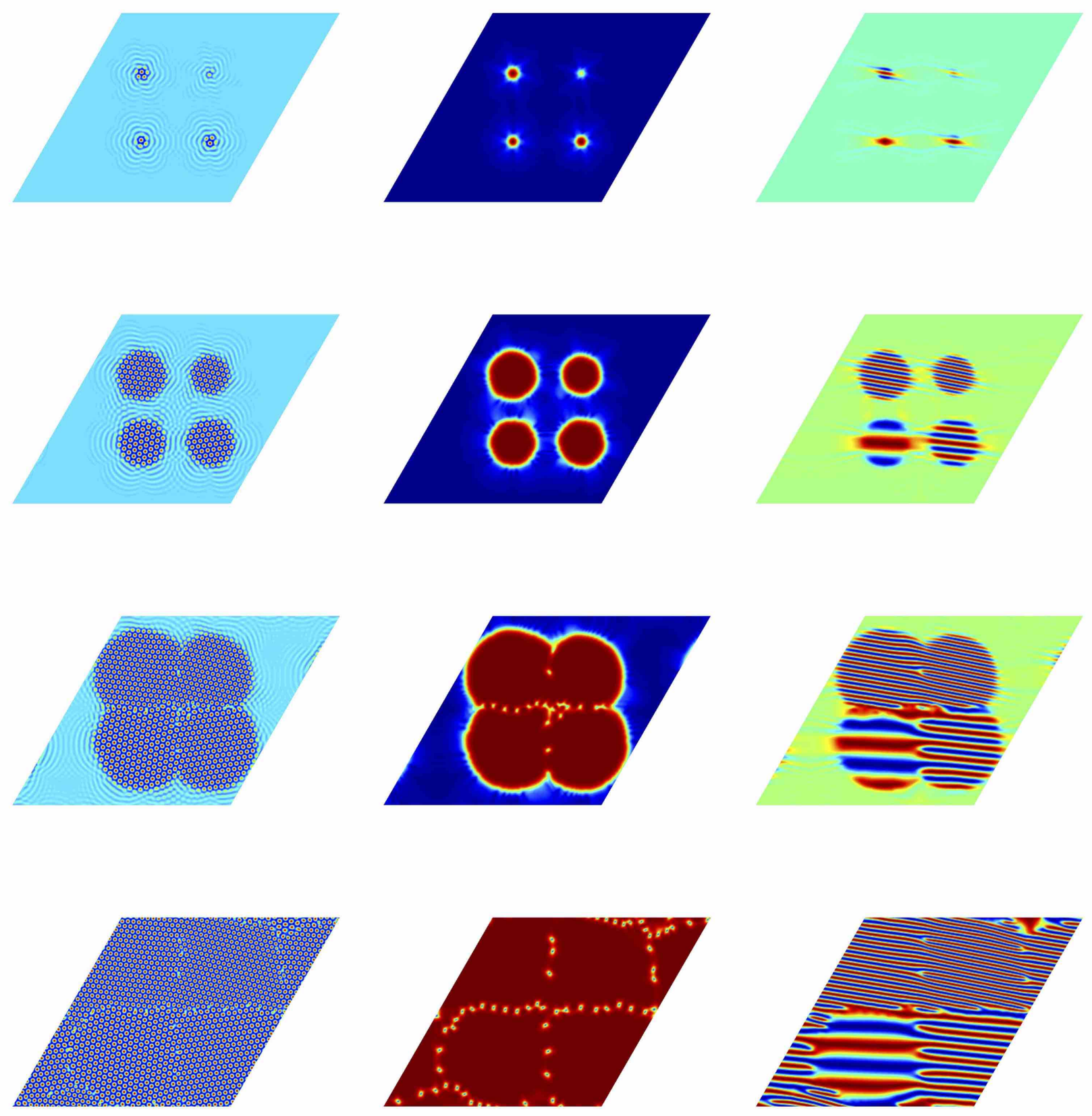

Figure 11: A simulation of crystal grain formation. Time is increasing from the top to the bottom. The parameters are given in the text. The first column shows the reconstructed density field $\rho$, obtained using (2.7). The second and third columns are plots of $\sum_{j=1}^{3}\left|\eta_{j}\right|$ and $u_{1}$, respectively. 
the similar result, the traditional PFC model requires much smaller grid size compared with the amplitude equations.

Remark 4.2. Adaptive time-stepping algorithms are an invaluable tool in the long time simulation of gradient flow processes, due to the existence of time-localized events featuring, for example, the merging or dissolution of interfaces or other structures coupled with the rapid reduction in energy. There have been quite a few numerical works of the adaptive time-stepping method in the existing literature, such as the ones for the Cahn-Hilliard equation [49], the molecular beam epitaxy (MBE) model [33], and the original PFC model [48], to name a few. The application of adaptive timestepping algorithms for the PFC amplitude model will also be considered in our future work.

\section{Conclusion and Future Work}

In this paper we constructed a first-order accurate in time, second-order accurate in space, unconditionally energy stable numerical scheme for 2D PFC amplitude expansion equations. In order to obtain a provable energy stable scheme, the problem was recast on a hexagonal domain with respect to the reciprocal lattice vectors. We analyzed the properties of the amplitude equations on this domain, and devised semi and fully discrete algorithms based on a hexagonal spatial mesh. We proved that our scheme is unconditionally uniquely solvable and unconditionally energy stable. To keep the paper as short as possible, a convergence analysis of the scheme was not provided, though this can be straightforwardly done given the energy stabilities that have been obtained. The stability and accuracy of the scheme was demonstrated numerically by several computational experiments, including grain rotations and crystal grain growth.

An efficient multi-grid solver was devised for this scheme - and is described briefly in the Appendix - but we did not explore the convergence analysis nor the practical efficiency of the solver in any detail. We plan to explore this aspect in more detail in a future work. Our immediate plans are to modify our scheme so that it can work for the case where the average density, $\rho_{0}$ is a variable in space, as discussed in [47]. This would allow us to explore more realistic simulations of melting and solidification. The convexity analysis is expected to easily extend to the variable average density case. We also plan to develop adaptive time-stepping schemes where the value of step size $s$ is adjusted at each time iteration to make the simulations more accurate. In addition, we also plan to develop a spatially adaptive version of the scheme using our adaptive finite difference/multigrid framework, BSAM [44].

\section{A Multigrid Solver for the Amplitude Equations}

In this section we discuss the details of an efficient, nonlinear multigrid solver. The main structure of the solver is borrowed from those reported in [25, 43]. We only modify it slightly to simulate amplitude equations. To simplify the writing a bit, let us define

$$
\begin{aligned}
& R_{1}:=2 \max \left\{\frac{\left(6 \nu \rho_{0}-2 \tau\right)^{2}}{3 \nu},\left(\frac{B_{0}}{2}+\frac{3 \nu \rho_{0}^{2}}{2}-\tau \rho_{0}\right)\right\} \\
& R_{2}:=2 \max \left\{0, \frac{\left(6 \nu \rho_{0}-2 \tau\right)^{2}}{3 \nu}-\left(\frac{B_{0}}{2}+\frac{3 \nu \rho_{0}^{2}}{2}-\tau \rho_{0}\right)\right\} .
\end{aligned}
$$


Then, our convex splitting scheme (3.54) - (3.57) may be expressed as follows: given $\boldsymbol{u}^{m}, \boldsymbol{v}^{m}, \boldsymbol{\kappa}^{m}, \boldsymbol{\zeta}^{m} \in$ $\left[C_{\mathrm{per}}^{\infty}(\mathrm{D})\right]^{3}$ find $\boldsymbol{u}, \boldsymbol{v}, \boldsymbol{\kappa}, \boldsymbol{\zeta} \in\left[C_{\mathrm{per}}^{\infty}(\mathrm{D})\right]^{3}$, whose components solve

$$
\begin{aligned}
u_{j}+ & s B_{4}\left(\Delta_{h} \kappa_{j}-4 a_{j} D_{j}\left(\zeta_{j}\right)-4 d_{j}\left(D_{j} u_{j}\right)\right) \\
& +s R_{1} u_{j}+6 s \nu A^{2} u_{j}+s\left(6 \nu \rho_{0}-2 \tau\right)\left(u_{\alpha_{j}} u_{\beta_{j}}-v_{\alpha_{j}} v_{\beta_{j}}\right) \\
= & s R_{2} u_{j}^{m}+3 s \nu\left(\left(u_{j}^{m}\right)^{2}+\left(v_{j}^{m}\right)^{2}\right) u_{j}^{m}+u_{j}^{m}, \\
\kappa_{j}= & \Delta_{h} u_{j}, \\
v_{j}+ & s B_{4}\left(\Delta_{h} \zeta_{j}+4 a_{j} D_{j}\left(\kappa_{j}\right)-4 d_{j}\left(D_{j} v_{j}\right)\right) \\
& +s R_{1} v_{j}+6 s \nu A^{2} v_{j}-s\left(6 \nu \rho_{0}-2 \tau\right)\left(u_{\alpha_{j}} v_{\beta_{j}}+v_{\alpha_{j}} u_{\beta_{j}}\right) \\
= & s R_{2} v_{j}^{m}+3 s \nu\left(\left(u_{j}^{m}\right)^{2}+\left(v_{j}^{m}\right)^{2}\right) v_{j}^{m}+v_{j}^{m}, \\
\zeta_{j}= & \Delta_{h} v_{j},
\end{aligned}
$$

for $j=1,2,3$. Observe that, for convenience, we have dropped the superscripted index $m+1$ for the unknown variables at the implicit time level. We have also represented the equations as a coupled system of 12 second-order finite difference equations. Now, we define operator and source terms as follows: for the operators, we set

$$
\begin{aligned}
N^{1, j}(\boldsymbol{u}, \boldsymbol{v}, \boldsymbol{\kappa}, \boldsymbol{\zeta}):= & u_{j}+s B_{4}\left(\Delta_{h} \kappa_{j}-4 a_{j} D_{j}\left(\zeta_{j}\right)-4 d_{j}\left(D_{j} u_{j}\right)\right) \\
& +s R_{1} u_{j}+6 s \nu A^{2} u_{j}+s\left(6 \nu \rho_{0}-2 \tau\right)\left(u_{\alpha_{j}} u_{\beta_{j}}-v_{\alpha_{j}} v_{\beta_{j}}\right), \\
N^{2, j}(\boldsymbol{u}, \boldsymbol{v}, \boldsymbol{\kappa}, \boldsymbol{\zeta}):= & \kappa_{j}-\Delta_{h} u_{j} \\
N^{3, j}(\boldsymbol{u}, \boldsymbol{v}, \boldsymbol{\kappa}, \boldsymbol{\zeta}):= & v_{j}+s B_{4}\left(\Delta_{h} \zeta_{j}+4 a_{j} D_{j}\left(\kappa_{j}\right)-4 d_{j}\left(D_{j} v_{j}\right)\right) \\
& +s R_{1} v_{j}+6 s \nu A^{2} v_{j}-s\left(6 \nu \rho_{0}-2 \tau\right)\left(u_{\alpha_{j}} v_{\beta_{j}}+v_{\alpha_{j}} u_{\beta_{j}}\right) \\
N^{4, j}(\boldsymbol{u}, \boldsymbol{v}, \boldsymbol{\kappa}, \boldsymbol{\zeta}):= & \zeta_{j}-\Delta_{h} v_{j} .
\end{aligned}
$$

For the source terms, we define

$$
\begin{aligned}
& S^{1, j}:=s R_{2} u_{j}^{m}+3 s \nu\left(\left(u_{j}^{m}\right)^{2}+\left(v_{j}^{m}\right)^{2}\right) u_{j}^{m}+u_{j}^{m}, \\
& S^{2, j}:=0 \\
& S^{3, j}:=s R_{2} v_{j}^{m}+3 s \nu\left(\left(u_{j}^{m}\right)^{2}+\left(v_{j}^{m}\right)^{2}\right) v_{j}^{m}+v_{j}^{m}, \\
& S^{4, j}:=0 .
\end{aligned}
$$

The scheme (A.3) - (A.6) is equivalent to $\mathbf{N}(\boldsymbol{u}, \boldsymbol{v}, \boldsymbol{\kappa}, \boldsymbol{\zeta})=\mathbf{S}$. Readers are referred to [43] for details of the FAS multigrid scheme. Here we only discuss the nonlinear smoothing scheme for the operator $N$, and the interpolation operators for communications between different grids.

For the smoothing process we use a nonlinear Gauss-Seidel method with Red-Black ordering. In what follows, to simplify the discussion, we give the details of the smoothing process using the simpler lexicographic ordering. Let $p$ be the index for the lexicographic Gauss-Seidel, and the smoothing scheme is constructed as follows: for every $(k, \ell)$, stepping lexicographically from $(1,1)$ 
to $(n, n)$, we solve the following linear systems:

$$
\begin{aligned}
u_{j, k, \ell}^{p+1}- & \frac{4 s B_{4}}{h^{2}} \kappa_{j, k, \ell}^{p+1}+6 s \nu\left(A_{k, \ell}^{p}\right)^{2} u_{j, k, \ell}^{p+1}+s R_{1} u_{j, k, \ell}^{p+1} \\
= & -\frac{2 s B_{4}}{3 h^{2}}\left(\kappa_{j, k+1, \ell}^{p}+\kappa_{j, k-1, \ell}^{p+1}+\kappa_{j, k, \ell+1}^{p}+\kappa_{j, k, \ell-1}^{p+1}+\kappa_{j, k-1, \ell+1}^{p}+\kappa_{j, k+1, \ell-1}^{p+1}\right) \\
& +4 s B_{4} a_{j} D_{j}\left(\zeta_{j, k, \ell}^{p}\right)+4 s B_{4} d_{j}\left(D_{j} u_{j, k, \ell}^{p}\right) \\
& -s\left(6 \nu \rho_{0}-2 \tau\right)\left(u_{\alpha_{j}, k, \ell}^{p} u_{\beta_{j}, k, \ell}^{p}-v_{\alpha_{j}, k, \ell}^{p} v_{\beta_{j}, k, \ell}^{p}\right)+S_{k, \ell}^{1, j}, \\
\kappa_{j, k, \ell}^{p+1}- & \frac{4}{h^{2}} u_{j, k, \ell}^{p+1} \\
= & \frac{2}{3 h^{2}}\left(u_{j, k+1, \ell}^{p}+u_{j, k-1, \ell}^{p+1}+u_{j, k, \ell+1}^{p}+u_{j, k, \ell-1}^{p+1}+u_{j, k-1, \ell+1}^{p}+u_{j, k+1, \ell-1}^{p+1}\right)+S_{k, \ell}^{2, j}, \\
v_{j, k, \ell}^{p+1}- & \frac{4 s B_{4}}{h^{2}} \zeta_{j, k, \ell}^{p+1}+6 s \nu\left(A_{k, \ell}^{p}\right)^{2} v_{j, k, \ell}^{p+1}+s R_{1} v_{j, k, \ell}^{p+1} \\
= & -\frac{2 s B_{4}}{3 h^{2}}\left(\zeta_{j, k+1, \ell}^{p}+\zeta_{j, k-1, \ell}^{p+1}+\zeta_{j, k, \ell+1}^{p}+\zeta_{j, k, \ell-1}^{p+1}+\zeta_{j, k-1, \ell+1}^{p}+\zeta_{j, k+1, \ell-1}^{p+1}\right) \\
& -4 s B_{4} a_{j} D_{j}\left(\kappa_{j, k, \ell}^{p}\right)+4 s B_{4} d_{j}\left(D_{j} v_{j, k, \ell}^{p}\right) \\
& +s\left(6 \nu \rho_{0}-2 \tau\right)\left(u_{\alpha_{j}, k, \ell}^{p} v_{\beta_{j}, k, \ell}^{p}+v_{\alpha_{j}, k, \ell}^{p} u_{\beta_{j}, k, \ell}^{p}\right)+S_{k, \ell}^{3, j}, \\
\zeta_{j, k, \ell}^{p+1}- & \frac{4}{h^{2}} v_{j, k, \ell}^{p+1} \\
= & \frac{2}{3 h^{2}}\left(v_{j, k+1, \ell}^{p}+v_{j, k-1, \ell}^{p+1}+v_{j, k, \ell+1}^{p}+v_{j, k, \ell-1}^{p+1}+v_{j, k-1, \ell+1}^{p}+v_{j, k+1, \ell-1}^{p+1}\right)+S_{k, \ell}^{4, j} .
\end{aligned}
$$

Note that we lag the quadratic nonlinear and directional (anisotropic) derivative terms to the $p^{\text {th }}$ iteration level. We use a local picard linearization on the cubic terms. For example,

$$
\left(A_{k, \ell}^{p+1}\right)^{2} u_{j, k, \ell}^{p+1} \approx\left(A_{k, \ell}^{p}\right)^{2} u_{j, k, \ell}^{p+1}
$$

Otherwise, this is a standard vector Gauss-Seidel smoothing. The linear system, which clearly decouples into six $2 \times 2$ linear sub-systems, is solved efficiently at each grid point using Cramer's rule.

Finally, since our hexagonal grid has the same logical structure as a square Cartesian grid, the standard prolongation and restriction operations can be utilized, as suggested in Figure. 12. Alternatively, we could use a weighted average that better respects the hexagonal symmetry of the problem, but we found that this was unnecessary.

\section{Acknowledgements}

VH acknowledges partial support from Academy of Finland through its COMP CoE Grants No. 251748 and 284621. JSL acknowledges partial support from NSF-CHE 1035218, NSF-DMR 1105409 and NSF-DMS 1217273. CW acknowledges partial support from NSF-DMS 1418689 and NSFC 11271281. SMW acknowledges partial support from NSF-DMS 1418692. SMW gratefully acknowledges Ken Elder for bring this problem to his attention, and for some initial discussions on the topic, during an IPAM workshop in 2012. 


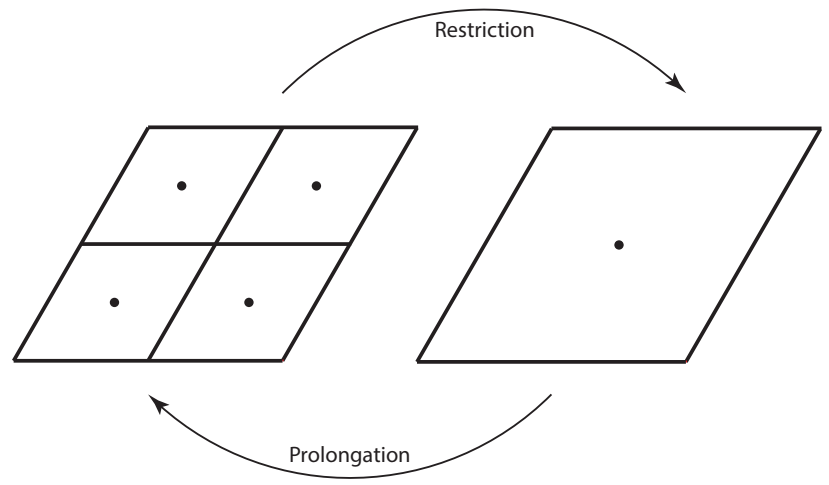

Figure 12: A schematic showing the prolongation and restriction operation on the hexagonal grid. We use simple four-point averaging for the restriction operation, and piecewise-constant interpolation for the prolongation operation. These operations are logically equivalent to those we use in the standard square Cartesian cell-centered mesh.

\section{References}

[1] C. Achim, M. Karttunen, K. R. Elder, E. Granato, T. Ala-Nissila, and S-C. Ying. Phase diagram and commensurate-incommensurate transitions in the phase field crystal model with an external pinning potential. Phys. Rev. E, 74:021104, 2006.

[2] C. Achim, J. Ramos, M. Karttunen, K. R. Elder, E. Granato, T. Ala-Nissila, and S-C. Ying. Nonlinear driven response of a phase-field crystal in a periodic pinning potential. Phys. Rev. E, 79:011606, 2009.

[3] B. Athreya, N. Goldenfeld, and J. Dantzig. Renormalization-group theory for the phase-field crystal equation. Phys. Rev. E, 74:011601, 2006.

[4] B. Athreya, N. Goldenfeld, J. Dantzig, M. Greenwood, and N. Provatas. Adaptive mesh computation of polycrystalline pattern formation using a renormalization-group reduction of the phase-field crystal model. Phys. Rev. E, 76:056706, 2007.

[5] A. Baskaran, Z. Hu, J. S. Lowengrub, C. Wang, S. M. Wise, and P. Zhou. Energy stable and efficient finite-difference nonlinear multigrid schemes for the modified phase field crystal equation. J. Comput. Phys., 250:270-292, 2013.

[6] A. Baskaran, J.S. Lowengrub, C. Wang, and S.M. Wise. Convergence analysis of a second order convex splitting scheme for the modified phase field crystal equation. SIAM J. Numer. Anal., 51:2851-2873, 2013.

[7] J. Berry, K. R. Elder, and M. Grant. Melting at dislocations and grain boundaries: A phase field crystal study. Phys. Rev. B, 77:061506, 2008.

[8] J. Berry, K. R. Elder, and M. Grant. Simulation of an atomistic dynamic field theory for monatomic liquis: Freezing and glass formation. Phys. Rev. E, 77:061506, 2008.

[9] J. Berry and M. Grant. Modeling multiple time scales during glass formation with phase-field crystals. Phys. Rev. Lett., 106:175702, 2011. 
[10] H. Cao and Z. Sun. Two finite difference schemes for the phase field crystal equation. Science China Mathematics, 58(11):2435-2454, 2015.

[11] K. R. Elder and M. Grant. Modeling elastic and plastic deformations in nonequilibrium processing using phase field crystals. Phys. Rev. E, 70:051605, 2004.

[12] K. R. Elder, M. Katakowski, M. Haataja, and M. Grant. Modeling Elasticity in Crystal Growth. Phys. Rev. Lett., 88(24):245701, 2002.

[13] K. R. Elder, N. Provatas, J. Berry, P. Stefanovic, and M. Grant. Phase-field crystal modeling and classical density functional theory of freezing. Phys. Rev. B, 75:064107, 2007.

[14] K. R. Elder, G. Rossi, P. Kanerva, F. Sanches, S-C. Ying, E. Granato, C. Achim, and T. AlaNissila. Patterning of heteroepitaxial overlayers from nano to micron scales. Phys. Rev. Lett., 108:226102, 2012.

[15] K. R. Elder, G. Rossi, P. Kanerva, F. Sanches, S-C. Ying, E. Granato, C. Achim, and T. AlaNissila. Modeling self-organization of thin strained metallic overlayers from atomic to micron scales. Phys. Rev. B, 88:075423, 2013.

[16] D. J. Eyre. Unconditionally gradient stable time marching the cahn-hilliard equation. In $M R S$ Proceedings, volume 529, page 39. Cambridge Univ Press, 1998.

[17] N. Goldenfeld, B. Athreya, and J. Dantzig. Renormalization group approach to multiscale simulation of polycrystalline materials using the phase field crystal model. Phys. Rev. E, 72:020601, 2005.

[18] N. Goldenfeld, B. Athreya, and J. Dantzig. Renormalization Group Approach to Multiscale Modelling in Materials Science. J. Stat. Phys., 125:1015-1023, 2006.

[19] H. Gomez and X. Nogueira. An unconditionally energy-stable method for the phase field crystal equation. Comput. Meth. Appl. Mech. Eng., 249(1):52?61, 2012.

[20] Z. Guan, C. Wang, and S. M. Wise. A convergent convex splitting scheme for the periodic nonlocal Cahn-Hilliard equation. Numer. Math., 128:377-406, 2014.

[21] R. Guo and Y. Xu. Local discontinuous galerkin method and high order semi-implicit scheme for the phase field crystal equation. SIAM J. Sci. Comput., 38(1):A105?A127, 2016.

[22] R. Heikes and D. A. Randall. Numerical integration of the shallow-water equations on a twisted icosahedral grid. part i: Basic design and results of tests. Mon. Weather Rev., 123:1862-1880, 1995.

[23] V. Heinonen, C. Achim, K. R. Elder, S. Buyukdagli, and T. Ala-Nissila. Phase-field-crystal models and mechanical equilibrium. Phys. Rev. E, 89:032411, 2014.

[24] T. Hirouchi, T. Takaki, and Y. Tomita. Development of numerical scheme for phase field crystal deformation simulation. Comput. Mater. Sci., 44:1192-1197, 2009.

[25] Z. Hu, S. M. Wise, C. Wang, and J. S. Lowengrub. Stable and efficient finite-difference nonlinear-multigrid schemes for the phase field crystal equation. J. Comput. Phys., 228:53235339, 2009. 
[26] Z-F. Huang and K. R. Elder. Mesoscopic and microscopic modeling of island formation in strain film epitaxy. Phys. Rev. Lett., 101:158701, 2008.

[27] Z-F. Huang and K. R. Elder. Morphological instability, evolution, and scaling in strained epitaxial films: An amplitude-equation analysis of the phase field crystal model. Phys. Rev. $B, 81: 364103,2010$.

[28] A. Jaatinen, C. Achim, K. R. Elder, and T. Ala-Nissila. Thermodynamics of bcc metals in phase-field-crystal models. Phys. Rev. E, 80:031602, 2009.

[29] D. Lee. Complete solution to seven-point schemes of discrete anisotropic laplacian on regular hexagons. Appl. Anal. Discrete Math., 9:180-197, 2015.

[30] J. Mellenthin, A. Karma, and M. Plapp. Phase-field crystal study of grain-boundary premelting. Phys. Rev. B, 78:184110, 2008.

[31] S. Nivckovic, M. B. Gavrilov, and I. A. Tovsic. Geostrophic adjustment on hexagonal grids. Mon. Weather Rev., 130:668-683, 2002.

[32] N. Provatas and K. Elder. Phase-Field Methods in Materials Science and Engineering. WileyVCH, Weinheim, Germany, 2010.

[33] Z. Qiao, Z. Zhang, and T. Tang. An adaptive time-stepping strategy for the molecular beam epitaxy models. SIAM J. Sci. Comput., 33:1395-1414, 2011.

[34] J. Ramos, E. Granato, C. Achim, S-C. Ying, K. R. Elder, and T. Ala-Nissila. Thermal fluctuations and phase diagrams of the phase-field crystal model with pinning. Phys. Rev. E, 78:031109, 2008.

[35] J. Ramos, E. Granato, S-C. Ying, C. Achim, K. R. Elder, and T. Ala-Nissila. Dynamical transitions and sliding friction of the phase-field-crystal model with pinning. Phys. Rev. E, 81:011121, 2010.

[36] R. Spatschek and A. Karma. Amplitude equations for polycrystalline materials with interaction between composition and stress. Phys. Rev. B, 81:214201, 2010.

[37] P. Stefanovic, M. Haataja, and N. Provatas. Phase-Field Crystals with Elastic Interactions. Phys. Rev. Lett., 96:225504, 2006.

[38] P. Stefanovic, M. Haataja, and N. Provatas. Phase field crystal study of deformation and plasticity in nanocrystalline materials. Phys. Rev. E, 80:046107, 2009.

[39] G. Tegze, G. I. Tóth, and L. Gránásy. Faceting and Branching in 2D Crystal Growth. Phys. Rev. Lett., 106:195502, 2011.

[40] H. Tomita, M. Tsugawa, M. Satoh, and K. Goto. Shallow water model on a modified icosahedral geodesic grid by using spring dynamics. J. Comput. Phys., 174:579-613, 2001.

[41] C. Wang, X. Wang, and S.M. Wise. Unconditionally stable schemes for equations of thin film epitaxy. Discrete Contin. Dyn. Sys. A, 28:405-423, 2010.

[42] C. Wang and S.M. Wise. An energy stable and convergent finite-difference scheme for the modified phase field crystal equation. SIAM J. Numer. Anal., 49:945-969, 2011. 
[43] S. M. Wise. Unconditionally stable finite difference, nonlinear multigrid simulation of the Cahn-Hilliard-Hele-Shaw system of equations. J. Sci. Comput., 44:38-68, 2010.

[44] S.M. Wise, J.S. Kim, and J.S. Lowengrub. Solving the regularized, strongly anisotropic CahnHilliard equation by an adaptive nonlinear multigrid method. J. Comput. Phys., 226:414-446, 2007.

[45] S.M. Wise, C. Wang, and J. Lowengrub. An energy stable and convergent finite-difference scheme for the phase field crystal equation. SIAM J. Numer. Anal., 47:2269-2288, 2009.

[46] K-A. Wu and P. W. Voorhees. Stress-induced morphological instabilities at the nanoscale examined using the phase field crystal approach. Phys. Rev. B, 80:125408, 2009.

[47] D-H. Yeon, Z-F. Huang, K. R. Elder, and K. Thornton. Density-amplitude formulation of the phase-field crystal model for two-phase coexistence in two and three dimensions. Philos. Mag., 90:237-263, 2010.

[48] Z. Zhang, Y. Ma, and Z. Qiao. An adaptive time-stepping strategy for solving the phase field crystal model. J. Comput. Phys., 249:204-215, 2013.

[49] Z. Zhang and Z. Qiao. An adaptive time-stepping strategy for the Cahn-Hilliard equation. Commun. Comput. Phys., 11:1261-1278, 2012.

[50] G. Zhou and S. R. Fulton. Fourier analysis of multigrid methods on hexagonal grids. SIAM J. Sci. Comput., 31:1518-1538, 2009. 